\title{
Eif2s3y Promotes the Proliferation of Spermatogonial Stem Cells by Activating ERK Signaling
}

\author{
Mengfei Zhang $\mathbb{D}^{1},{ }^{1}$ Na Li $\mathbb{D}^{1},{ }^{1}$ Wenqing Liu $\mathbb{D}^{1},{ }^{1}$ Xiaomin Du ${ }^{\mathbb{D}},{ }^{1}$ Yudong Wei $\mathbb{D}^{1}{ }^{1}$ \\ Donghui Yang $\odot,{ }^{1}$ Zhe Zhou, ${ }^{1}$ Fanglin Ma $₫$, ${ }^{1}$ Sha Peng, ${ }^{1}$ Shiqiang Zhang, ${ }^{1}$ Xin He, \\ Chunling Bai, ${ }^{2}$ Guangpeng Li $\left(\mathbb{1},{ }^{2}\right.$ and Jinlian Hua $\mathbb{1}^{1}$ \\ ${ }^{1}$ College of Veterinary Medicine, Shaanxi Centre of Stem Cells Engineering \& Technology, Northwest A\&F University, Yangling, \\ Shaanxi 712100, China \\ ${ }^{2}$ State Key Laboratory of Reproductive Regulation and Breeding of Grassland Livestock, School of Life Sciences, \\ Inner Mongolia University, Hohhot 010070, China
}

Correspondence should be addressed to Jinlian Hua; jinlianhua@nwsuaf.edu.cn

Received 8 October 2020; Revised 9 January 2021; Accepted 12 January 2021; Published 30 January 2021

Academic Editor: Valeria Sorrenti

Copyright (c) 2021 Mengfei Zhang et al. This is an open access article distributed under the Creative Commons Attribution License, which permits unrestricted use, distribution, and reproduction in any medium, provided the original work is properly cited.

\begin{abstract}
The future fertility of males with cancer may be irreversibly compromised by chemotherapy and/or radiotherapy. Spermatogonial stem cell transplantation is believed to be a way to restore fertility in men. However, the survival efficiency of transplanted cells is still low. Eukaryotic translation initiation factor 2 subunit 3 and structural gene Y-linked (Eif2s3y) located on the Y chromosome of male animals is a coding gene of eIF $2 \gamma$ which mainly functions in translation initiation. Recently, the emerging role of Eif2s $3 y$ in spermatogenesis has been emphasized in several studies. However, the underlying mechanism is still unclear. In addition, how Eif2s3y functions in large animals remains largely unknown. In this study, we obtained the CDS sequence of the Eif2s3y gene from the testis of dairy goats and found that this gene was highly expressed in the testis and was evolutionarily conserved among different species. Interestingly, overexpression of Eif2s $3 y$ promoted the proliferation of spermatogonial stem cells of dairy goats by activating the ERK signaling pathway. In animal experiments, overexpressing Eif2s3y promoted transplanted goat spermatogonial stem cells and produced more colonies after microinjection into the seminiferous tubules of infertile mice. In conclusion, our study highlights an undiscovered role of Eif2s $3 y$ in dairy goat reproduction. This finding may provide an important basis for future works regarding male spermatogenic cell restoration and represent a major advance toward surrogate sires becoming a tool for disseminating and regenerating germplasm in all mammals.
\end{abstract}

\section{Introduction}

Spermatogenesis is essential for the continuation of most species. The reduction of spermatogonial stem cells (SSCs) can destroy spermatogenesis and leads to male infertility [1, 2]. In addition to maintaining stable spermatogenesis, studies in mice have shown that a small fraction of undifferentiated spermatogonia can regenerate spermatogenic lineage after being isolated from donor tissues and transplanted into the testis of recipient males lacking endogenous reproductive lines [3]. These regenerated spermatogonia are often referred to as spermatogonial stem cells. SSCs are located on the basement membrane of seminiferous tubules, and the delicate control of SSC self-renewal and differentiation critically determines sperm production in male animals [2, 4]. Therefore, a defect in SSC proliferation usually results in reduced germ cell number or even male infertility [5].

Chemotherapeutic drugs, such as busulfan and cisplatin, cause male reproductive damage and long-term infertility by damaging SSCs $[6,7]$. In human reproductive medicine, SSCs can be used to solve infertility caused by spermatogenesis and maturation disorders [8]. Spermatogonial stem cell transplantation (SSCT) has many potential applications and may have a significant impact on society. Successful spermatogenesis has not been achieved following the transplantation of human testis tissue. However, there have been 
successful cases of animal SSCT, such as mice, dogs, and nonhuman primates $[9,10]$. Thus, improving the proliferation ability of SSCs is critical for the rapid restoration of male reproductive capacity.

Eukaryotic translation initiation factor 2 subunit 3 and structural gene Y-linked (Eif2s3y) is located on the Y chromosome of male animals and is traditionally considered to be involved in the formation of the eIF2 polymer to mediate translation initiation $[11,12]$. In recent years, several studies have shown that Eif2s $3 y$ is essential for mouse spermatogenesis $[13,14]$. In 2014, Yamauchi et al. reported that mouse progeny could be generated by male germ cells with the $\mathrm{Y}$ chromosome contribution limited to only two genes, Sry and Eif2s3y $[15,16]$. Importantly, Eif2s $3 y$ may be the only $\mathrm{Y}$ chromosome gene required to drive mouse spermatogenesis. In our previous studies, increased efficiency of haploid cell induction has been detected in Eif2s3y-overexpressing (oeEif2s3y) embryonic stem cells (ESCs) [17]. However, how Eif $2 s 3 y$ improves the efficiency of spermatogenesis is still unclear.

In the present study, we wanted to explore the role and regulatory mechanism of Eif $2 s 3 y$ in dairy goats. We obtained the Eif2s3y gene fragment of dairy goats and found that the expression level of Eif2s3y in the testis was significantly higher than that in other tissues. In addition, we found that Eif $2 s 3 y$ promoted goat SSC proliferation dependent on the extracellular regulated protein kinases (ERK) signaling pathway. The SSCT experiment showed that Eif2s3y could increase the number of SSCs transplanted into busulfantreated mice. Our study may provide an efficient approach for the repair of male spermatogenic cells in large animals and improve the efficiency of livestock genetic breeding in the future.

\section{Materials and Methods}

2.1. Animal Experiments. All animal experiments were performed in accordance with the Guide for the Care and Use of Laboratory Animals (Ministry of Science and Technology of the People's Republic of China, Policy No. 2006 398) and were approved by the Animal Care and Use Center of the Northwest A\&F University.

Different tissues and testes at different ages $(1,3,6,9,12$, 18 , and 24 months) of Guanzhong dairy goats were supplied by Yaoan slaughterhouse in the Yangling Agricultural Hightech Industrial Demonstration Zone. Three male goats from each age were used in the testis collection. These tissues were then used to extract RNA by using RNAiso Plus (\#9109, Takara Bio Inc., Japan).

The male ICR mice used for the infertile mouse model were purchased from Dashuo Laboratory Animal Limited Company in Chengdu, China. Twenty 7-week-old male mice were treated with busulfan (B2635-25G, Sigma-Aldrich by Merck) at a dose of $30 \mathrm{mg} / \mathrm{kg}$ for 2 weeks to be rendered infertile. These busulfan-treated mice were used for spermatogonial transplantation $[1,18]$.

2.2. Cell Culture and Preparation of Dairy Goat SSCs. The procedures for isolating and purifying SSCs were in accor- dance with a previous study, and the morphology and function of SSCs we used have been verified [19-21]. The procedures for isolating and purifying SSCs are as follows. Testes from dairy goats of 3 months were aseptically collected. After washing five times with phosphate-buffered saline (PBS) containing $100 \mathrm{U} / \mathrm{mL}$ penicillin and $100 \mathrm{mg} / \mathrm{mL}$ streptomycin, testes were cut into small pieces by using sterile scissors. Seminiferous epithelial cells were incubated with an enzyme cocktail containing $0.1 \%$ collagenase IV (Invitrogen) and $10 \mu \mathrm{g} / \mathrm{mL}$ DNase I (Sigma-Aldrich by Merck) at $37^{\circ} \mathrm{C}$ for $30 \mathrm{~min}$, and the cell suspension was blended every $10 \mathrm{~min}$ at the same time. The dissociated fragments were then digested with $0.25 \%$ trypsin (Invitrogen) for $15 \mathrm{~min}$, followed by neutralization with Dulbecco's modified Eagle's medium (DMEM, Invitrogen, Carlsbad, CA, USA) containing 10\% FBS (Gibco, MA, USA). The cell suspension was then filtered by $40 \mu \mathrm{m}$ copper meshes to exclude the seminiferous tubules. Then, the cell suspension was plated in culture dishes and incubated in an atmosphere composed of $5 \% \mathrm{CO}_{2}$ at $37^{\circ} \mathrm{C}$ for 2 hours.

Nonadherent SSCs were obtained and removed to a new dish when the Leydig cells attached to the culture dish. Then, these cells were purified by the MASC technique to obtain Thy1-positive cells. Dairy goat SSCs were cultured in a medium containing DMEM/F12 (Invitrogen) with 1\% FBS, $10 \%$ KSR (Invitrogen), $0.1 \mathrm{mM} \beta$-mercaptoethanol (SigmaAldrich by Merck), 1\% nonessential amino acids (Invitrogen), 1\% L-glutamine (Invitrogen), $10 \mathrm{ng} / \mathrm{mL}$ basic fibroblast growth factor (bFGF, Millipore), $10 \mathrm{ng} / \mathrm{mL}$ GDNF (Reproach), $50 \mathrm{ng} / \mathrm{mL}$ Gfral (Sino Biological, Inc., Beijing, China), and $20 \mathrm{ng} / \mathrm{mL}$ epidermal growth factor (EGF, Sino Biological, Inc.) [22, 23]. These cells were cultured for 12 hours at $37^{\circ} \mathrm{C}$, supplemented with $5 \% \mathrm{CO}_{2}$ in the air. The medium was refreshed every day. The dairy goat SSCs were passaged by TrypLE (Invitrogen).

2.3. Seminiferous Tubule Transplantation. For SSCT, approximately $100 \mu \mathrm{L}$ of a single cell suspension or medium was injected through the efferent duct into the left testis or right testis of busulfan-treated mice, respectively. The testis which was injected with the medium was the control group. The seminiferous tubule injection protocol was conducted as previously reported $[24,25]$. These testes were collected for analysis 4 weeks after injection [26].

2.4. Construction of Recombination Plasmid. The primer sequences for the dairy goat Eif2s3y CDS clone which were designed according to the published Mus musculus Eif2s3y mRNA sequence (XM_006531609) were as follows: forward: 5'-AGAATTCTTCGGCAAGATGGCG-3', reverse: $5^{\prime}$ -AGCGGCCGCCTTCATTCATCATC-3'.

Eif $2 s 3 y$ was amplified from the dairy goat testicular cDNA by a reverse transcription-polymerase chain reaction. Then, the specific fragments were cloned into the pCDHCMV-MCS-EF1 vector. Nucleotide fragments for knocking down experiments which were sent to biological companies for synthesis were as follows: 5 '-CCGGGAACAGATACTT GCATTTGTACTCGAGTACAAATGCAAGTATCTGTT CTTTTTTG-3'. The specific nucleotide fragments were 
cloned into the CD513B-U6-shEif2s3y vector. The recombinant plasmid pCDH-CMV-Eif2s3y-EF1-puro (oeEif2s $3 y$ ), CD513B-U6-shEif2s3y (shEif2s3y), and assistant plasmids PAX2 and VSVG were stored in Shaanxi Centre of Stem Cells Engineering \& Technology, Northwest A\&F University [27].

2.5. Lentivirus Preparation and Infection. Lentivirus production was described previously [28]. Assistant plasmids PAX2 and VSVG were cotransfected with pCDH-CMV-Eif2s3yEF1-puro or CD513B-U6-shEif2s3y in HEK293T cells. The oeEif2s $3 y$ or shEif2s $3 y$ lentivirus was collected 48 hours later after substituted. The primary SSCs were infected with lentivirus oeEif2s3y or shEif2s3y when the density reached $80 \%$ complementing with polybrene (Sigma-Aldrich by Merck) to increase transfection efficiency. The infected SSCs were then cultured with a medium containing $500 \mathrm{ng} / \mathrm{mL}$ puromycin (Sigma-Aldrich by Merck) for 1 week in order to increase the proportion of positive cells.

2.6. Ethynyl-Deoxyuridine (EdU) Incorporation Assay. EdU incorporation assay was performed as per the manufacturer's instructions (C10310-1, RiboBio, Guangzhou, China). SSCs planted in a 48 -well plate were incubated with the $50 \mu \mathrm{M}$ EdU medium for $2 \mathrm{~h}$. Then, the EdU medium was discarded. Cells were fixed with $4 \%$ paraformaldehyde at room temperature for $15 \mathrm{~min}$ and decolorized in $2 \mathrm{mg} / \mathrm{mL}$ glycine for $10 \mathrm{~min}$. After washing with PBS, cells were permeated by $0.5 \%$ Triton X-100 for $10 \mathrm{~min}$. The staining buffer was added and incubated in the dark at room temperature for $30 \mathrm{~min}$. After washing with PBS, the nuclei were visualized by Hoechst 33342 (Sigma-Aldrich by Merck). The cells were washed three times and observed under a fluorescence microscope.

Three culture wells were used in each group. At least three cell images and 300 cells per well were taken randomly. The ratio of the number of red fluorescent cells to the number of blue fluorescent cells is the ratio of positive cells [28]. The proportion of positive cells is positively correlated with the cell proliferation rate.

\subsection{Quantitative Reverse Transcription-Polymerase Chain} Reaction ( $q R T-P C R$ ) Analysis. The qRT-PCR analysis was in accordance with a previous report [17]. Tissues and cells were harvested at the proper time, and total RNAs were extracted using the TRIzol reagent (RNAiso Plus, \#9109, Takara Bio Inc., Japan). RNA integrity was analyzed by agarose gel electrophoresis, and the concentration was determined using a NanoDrop 2000 Spectrophotometer (Thermo Fisher Scientific, USA). Reverse transcription was performed using the RevertAid First Strand cDNA Synthesis Kit (Lot 00887496, Thermo Fisher Scientific, Waltham, Massachusetts, USA). Aliquots of undiluted cDNA were stored at $-20^{\circ} \mathrm{C}$ and used for RT-PCR and real-time PCR. RT-qPCR was conducted on a CFX Connect Real-Time System (BioRad, California, USA) using the SYBR Premix Real-Time PCR Kit (FP215-01, Tiangen Biotech, Beijing, China) in accordance with the manufacturer's instructions. The expression levels of mRNAs were normalized to GAPDH and $\beta$ actin. The qRT-PCR primers used in this article are listed in Supplemental Table 1. All primer sequences were determined through established GenBank sequences. The PCR efficiency was evaluated and analyzed by agarose gel electrophoresis.

2.8. Immunofluorescence (IF) Staining. Immunofluorescence staining of testes and SSCs was conducted as previously reported [27]. The primary antibodies used in this study are listed as follows: rabbit anti-eIF2 $\gamma$ (1:200; PA5-31177, Thermo Fisher Scientific, MA, USA), rabbit anti-DDX4 ( $1: 200$; ab13840, Abcam, Cambridge, UK), mouse antiZBTB16 (1:200; sc-28319, Santa Cruz Biotechnology, CA, USA), rabbit anti-STRA8 $(1: 200$; ab49602, Abcam, Cambridge, UK), mouse anti-GFRa1 (1:200; sc-271546, Santa Cruz Biotechnology, CA, USA), rabbit anti-SOX9 (1:200; ab185230, Abcam, Cambridge, UK), and rabbit anti-StAR ( $1: 100$; bs-20388R, Bioss, Beijing, China). Secondary antibodies are as follows: Alexa Fluor 488-goat anti-rabbit IgG (1:400; ZF-0511, ZSGB-BIO, Beijing, China) and Alexa Fluor 568-goat anti-mouse (1:400; ZF-0513, ZSGB-BIO, Beijing, China).

2.9. Population Doubling Time (PDT) Determination. The PDT of dairy goat SSCs was estimated according to the protocol described previously [29]. Briefly, cells were serially subcultured; the initial seeding cell number and the total cell number cultured $24 \mathrm{~h}$ later were all counted, respectively. PDT was calculated according to the formula PDT $=\left[\log _{2} /(\right.$ $\left.\left.\log N_{t}-\log N_{0}\right)\right] \times t$, where $N_{0}$ means the number of seeded cells, $N_{t}$ indicates the number of cells after $t(\mathrm{~h})$ of culturing, and $t$ means the duration of cell culturing hours.

2.10. Western Blotting. Western blotting (WB) was estimated according to a previous article [27]. The antibodies used in this study are listed as follows: anti-eIF2 $\gamma$ (1:500; PA531177, Thermo Fisher Scientific, MA, USA), anti-PCNA (1:500; BM0104, Boster, Wuhan, China), anti-Cyclin D (1:500; WL01435a, Wanlei, Shenyang, China), antiGAPDH (1:2000; AC002, ABclonal, Wuhan, China), antiZBTB16 (1:500; No. D222893, BBI Life, Shanghai, China), anti-pERK $(1: 2000$; \#4370, CST, Boston, USA), and antiERK (1:2000; \#9194, CST, Boston, USA). The results were detected using a Bio-Rad imaging system (Bio-Rad, Hercules, CA, USA) and quantified using ImageJ (V1.48d).

2.11. Bioinformatics Analysis. The dairy goat Eif2s3y CDS was sequenced by Sangon, China. Multiple sequence alignment among different species was performed by DNAMAN software, and the phylogenetic tree was depicted with MEGA 4.1. The amino acid sequences of Eif2s3y proteins in different species were also analyzed by DNAMAN software. The protein secondary structure was predicted by DNAStar software. The domains contained in Eif2s $3 y$ protein were predicted by the SWISS-MODEL Workspace website and RasMol software [30].

2.12. ERK Pathway Inhibitor and Activator. To confirm the function of ERK signaling in Eif2s3y regulation, SSCs were treated with $1 \mu \mathrm{M}$ ERK pathway inhibitor PD0325901 (APExBIO Technology LLC, A3013, Houston, USA) or 


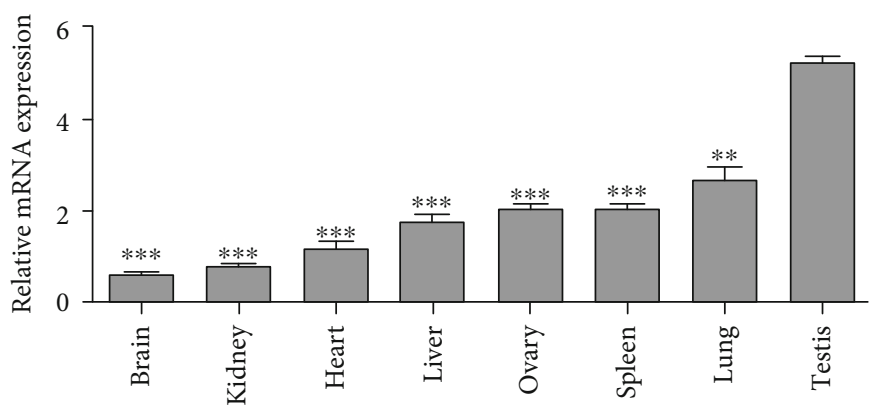

(a)

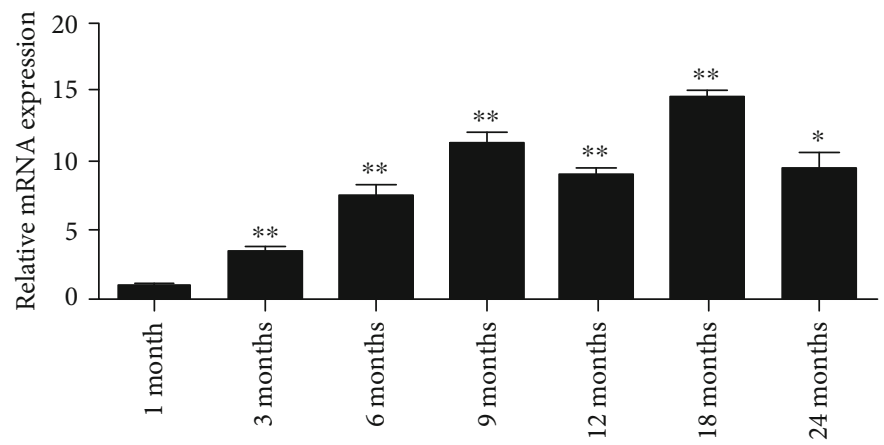

(b)

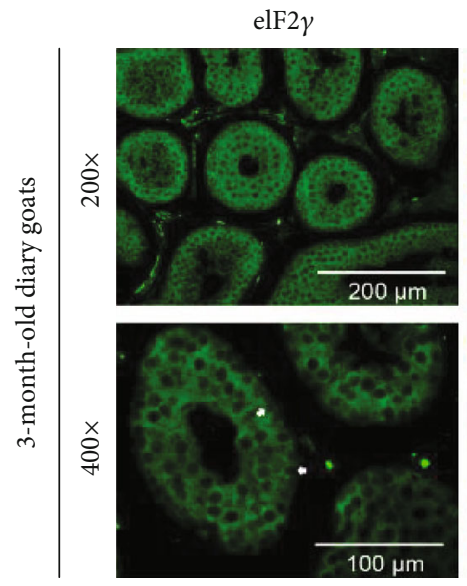

Hoechst 33342
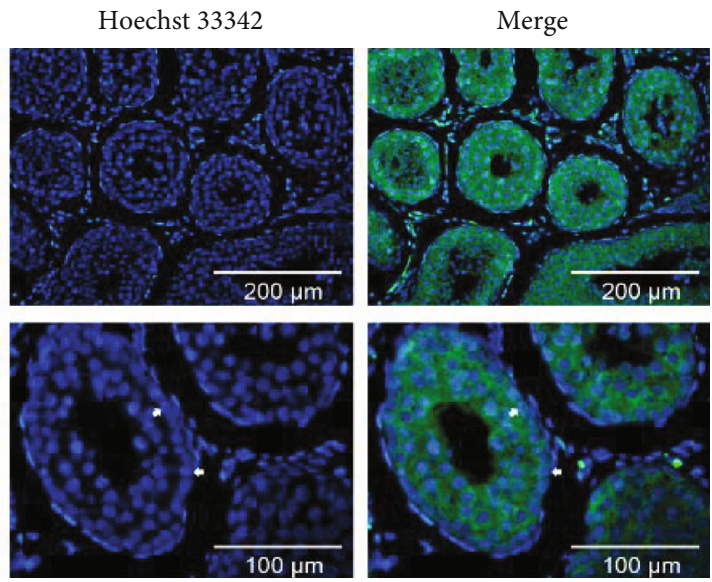

(c)

Figure 1: Continued. 

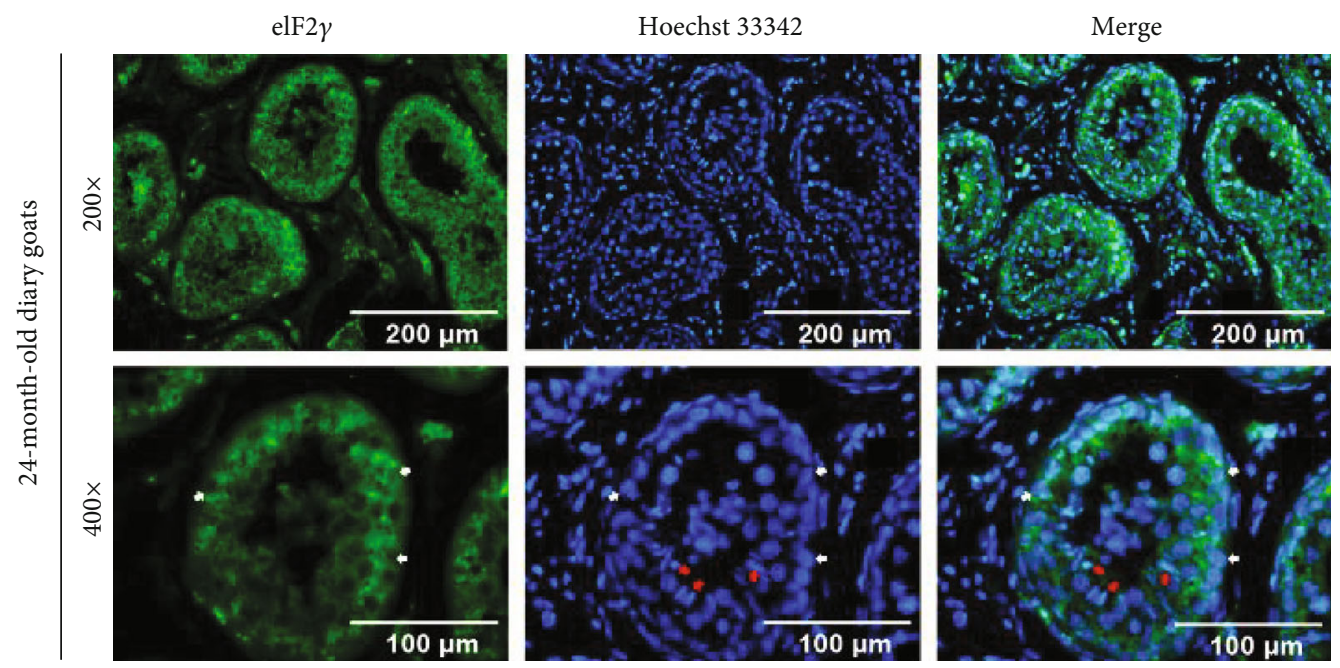

(d)

FIGURE 1: The expression pattern of Eif2s3y in dairy goats. (a) Real-time PCR analysis of Eif2s3y expression levels in different tissues of adult dairy goats. All results were compared with the testis. (b) Real-time PCR analysis of Eif2s3y expression levels in the testes of dairy goats of different ages. All results were compared with that of 1-month-old goats. (c) Immunofluorescence staining of eIF2 $\gamma$ in the testes of 3month-old dairy goats counterstained with Hoechst 33342. The white arrows indicated typical SSCs. Scale bars, $200 \mu \mathrm{m}$ (up) and $100 \mu \mathrm{m}$ (down). (d) Immunofluorescence staining of eIF2 $\gamma$ in the testes of 24-month-old dairy goats counterstained with Hoechst 33342 . The white arrows indicated typical SSCs, and the red arrows indicated typical sperms. Scale bars, $200 \mu \mathrm{m}$ (up) and $100 \mu \mathrm{m}$ (down). Data are presented as mean $\pm \mathrm{SD}$ and are represented by three independent repetitions; ${ }^{*} P<0.05,{ }^{* *} P<0.01$, and ${ }^{* * *} P<0.001$.

$10 \mu \mathrm{M}$ ERK pathway activator 12-O-tetradecanoylphorbol13-acetate (TPA, APExBIO Technology LLC, N2060, Houston, USA) for $24 \mathrm{~h}$, respectively [31]. PD0325901 effectively inhibits the phosphorylation of ERK1/2 in multiple cell lines, while TPA activates the phosphorylation of ERK1/2 [32]. There were three replicates in each group of cells. The diluent of the reagent is DMSO.

2.13. Statistical Analysis. Relative gene expression was analyzed by the comparative $\mathrm{Ct}$ method ( $2^{-\Delta \Delta \mathrm{Ct}}$ method). To compare significant differences, a two-tailed Student's $t$-test was used. The results were represented as mean \pm SD. All results were replicated at least 3 times. Statistical analyses were analyzed by SPSS 20.0 software and GraphPad Prism software (La Jolla, CA). $P$ values $<0.05$ were considered statistically significant $\left({ }^{*} P<0.05,{ }^{* *} P<0.01\right.$, and $\left.{ }^{* * *} P<0.001\right)$.

\section{Results}

3.1. The Expression Pattern of Eif2s3y in Dairy Goats. First, we performed qRT-PCR to clarify the expression pattern of Eif $2 s 3 y$ in dairy goats. The results showed that Eif $2 s 3 y$ was widely expressed in different tissues including the brain, kidney, heart, liver, ovary, spleen, lung, and testis (Figure 1(a)). Of note, the testis showed the highest expression level of Eif $2 s 3 y(P<0.01)$ and this level tended to increase gradually over time $(P<0.05)$, and it sustained high levels after sexual maturity than before (Figure 1(b)). Then, immunofluorescence staining was performed to analyze the expression pattern of eIF $2 \gamma$ in the testes of 3- and 24-month-old goats. The result showed that Eif2s3y could be expressed in SSCs, Sertoli cells, and Leydig cells (Figure 1(c); Supplemental
Fig. 2A and B). Of note, lots of sperms could be observed in the testes of 24-month-old goats while not in those of 3month-old goats (Figure 1(d); Supplemental Fig. 2C). We could see from these results that Eif2s3y was highly expressed in testes and eIF $2 \gamma$ protein mainly existed in the cytoplasm. The high expression of Eif2s3y in spermatogonia made us want to study their function in these cells.

3.2. Structure and Bioinformatics Analysis of Eif2s3y in Dairy Goats. A pair of specific cloning primers for the CDS region of the dairy goat Eif2s $3 y$ gene was designed as described in Materials and Methods. We further cloned the Eif2s3y gene of dairy goats by PCR, and three repetitions were made (Figure 2(a)). The fragments whose sizes were between $1000 \mathrm{bp}$ and $2000 \mathrm{bp}$ were considered to be the goat Eif2s3y gene. Next, we inserted the gene into the pMD18-T vector for sequencing analysis, which showed that the size of the CDS region of the dairy goat Eif2s3y gene was $1413 \mathrm{bp}$. We uploaded the sequence information to the National Center for Biotechnology Information (NCBI) and obtained a formal gene serial number (GenBank: KP326346.1).

Eif2s3y gene sequences of Homo sapiens, Microcebus murinus, Capra hircus, Bos taurus, Rattus norvegicus, Mus musculus, Tokudaia osimensis, Loxodonta africana, and Xenopus tropicalis obtained from NCBI indicated that this gene was widely expressed in different species (Figure 2(b)). We analyzed the phylogenetic tree of Eif2s3y and compared their nucleotide and amino acid sequences (Figure 2(b)). The results showed a $97.98 \%$ similarity for amino acid among different species and suggested that Eif2s3y was highly conserved among different species (Figure 2(c)). Then, we predicted the protein structure of Eif2s3y through SWISS- 


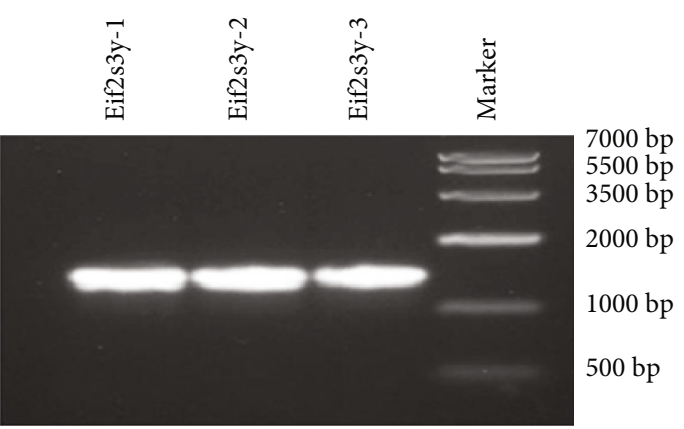

(a)

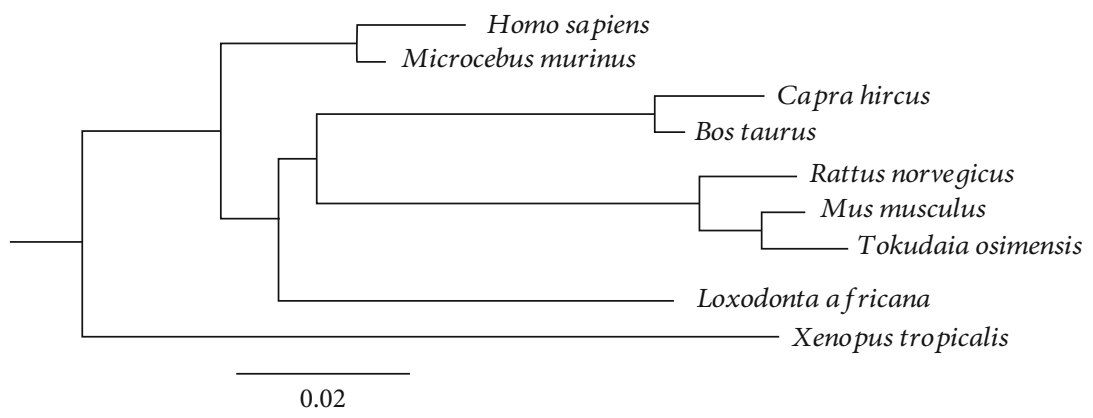

(b)

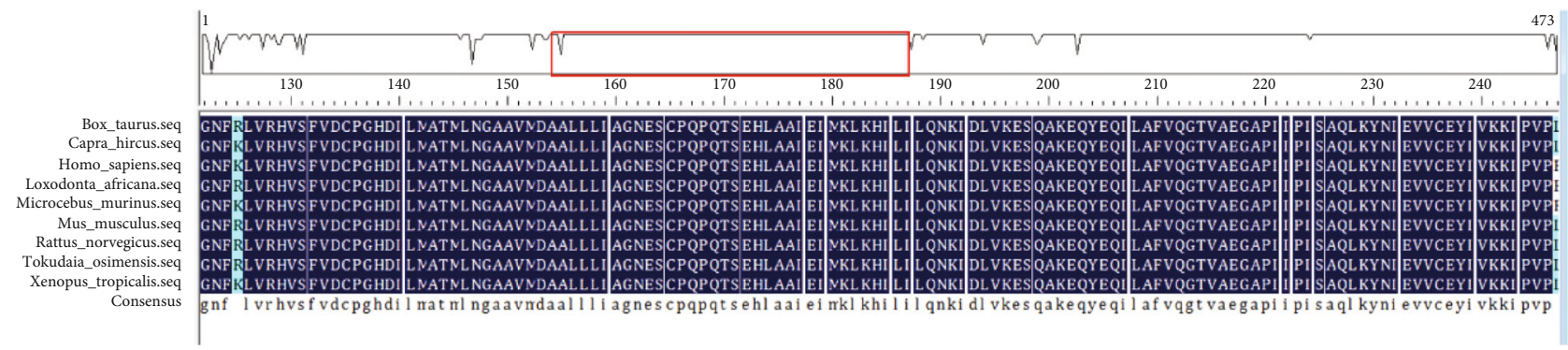

(c)

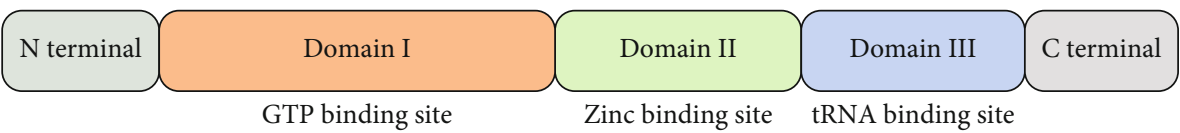

(d)
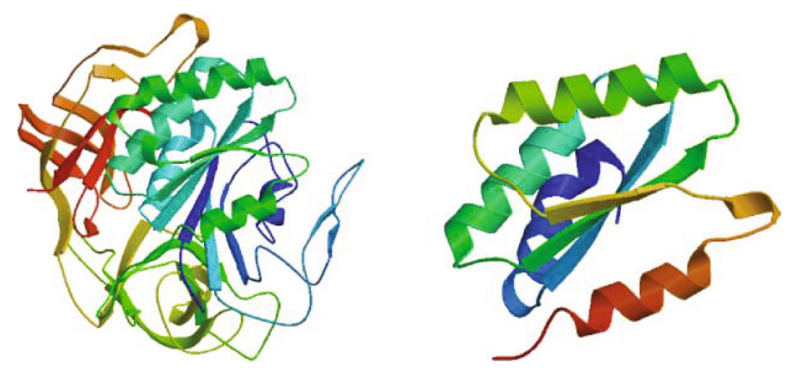

(e)

FIGURE 2: Structure and bioinformatics analysis of Eif2s3y. (a) PCR analysis of Eif2s $3 y$ in dairy goat testes. Three independent duplications all had the same size of $1413 \mathrm{bp}$. (b) Eif2s3y phylogenetic tree in different species constructed by MEGA 4.1 software. (c) Eif2s3y amino acid sequence alignment of different species analyzed by DNAMAN software. (d) Conserved domains of Capra hircus Eif2s3y CDS were predicted by NCBI CD-Search. Some important structures were found in conserved domains. (e) Eif2 $\gamma$ protein structural pattern (up) and four zinc finger domains (down) analyzed by the SWISS-MODEL Workspace website.

MODEL Workspace and found an important binding region comprising four tandem zinc finger domains (Figure 2(e)). Another prediction gave a schematic map of protein domain analysis of Eif2 $\gamma$ by NCBI CD-Search (Figures 2(d) and 2(e)). These results indicated that Eif2s $3 y$ was conserved and might have similar functions in different species. 


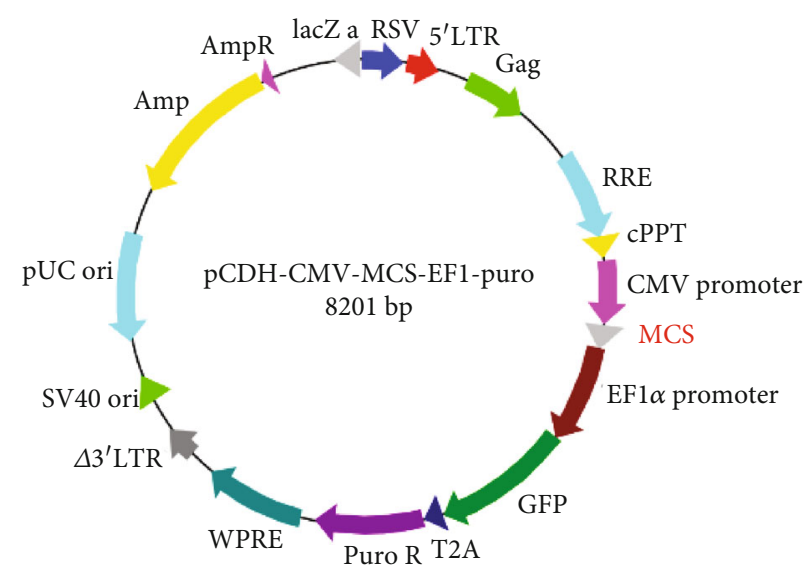

(a)
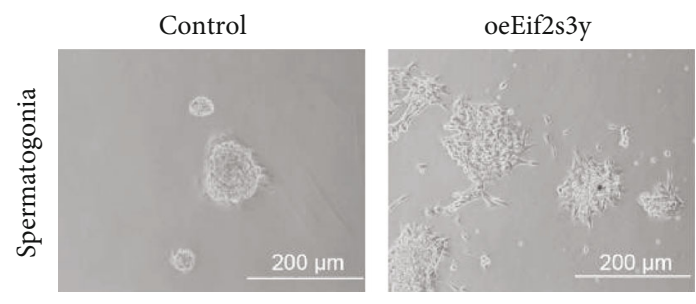

(c)
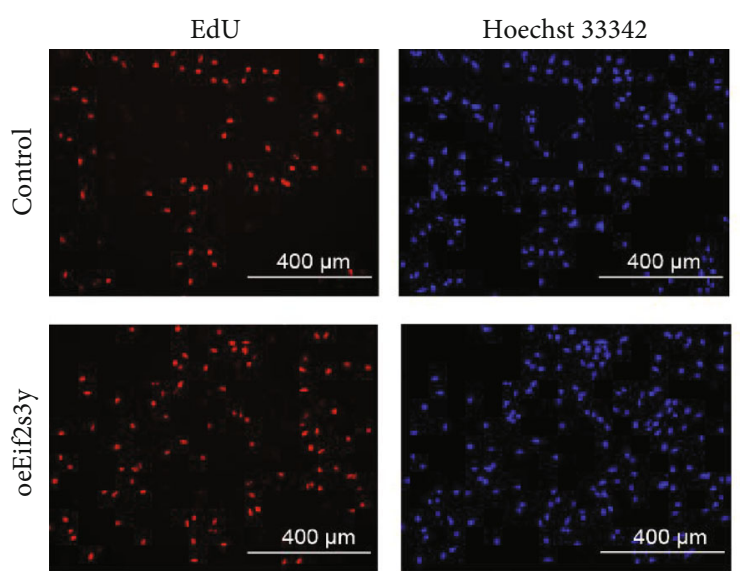

(e)

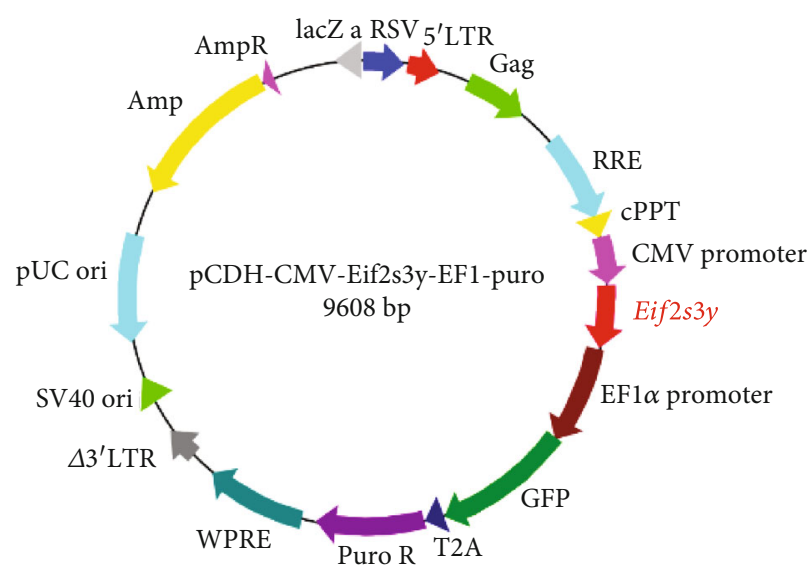

(b)

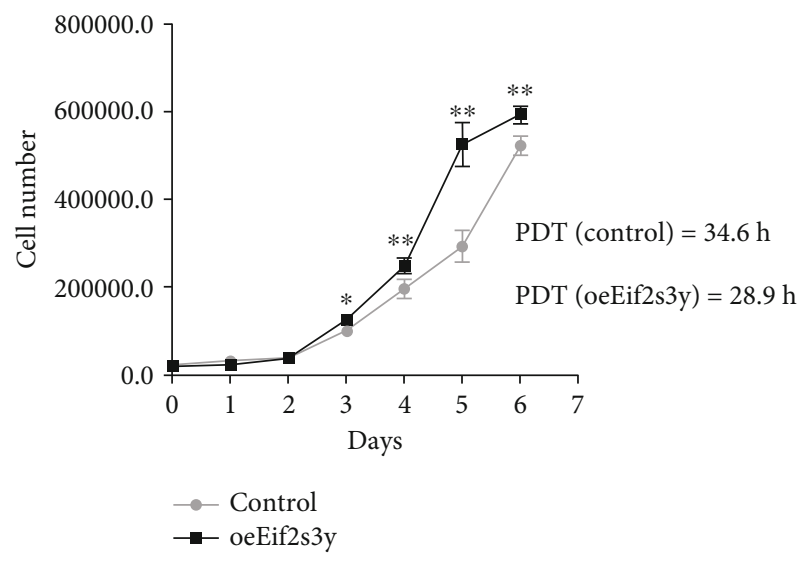

(d)
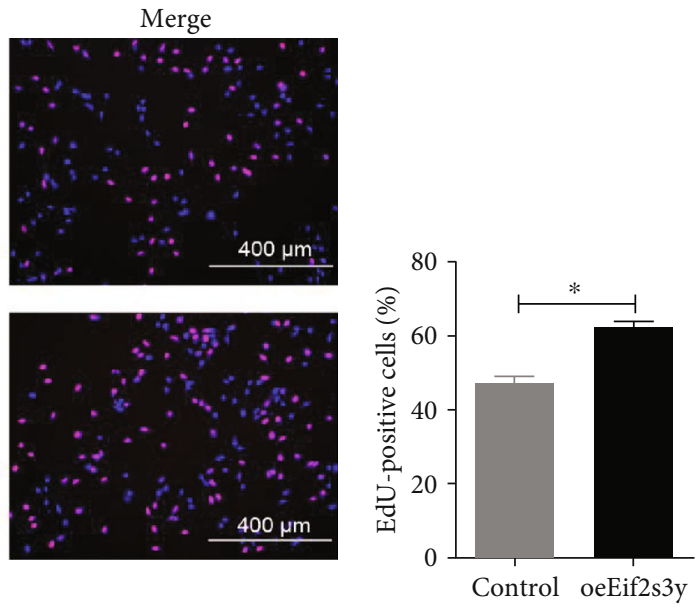

(f)

Figure 3: Continued. 


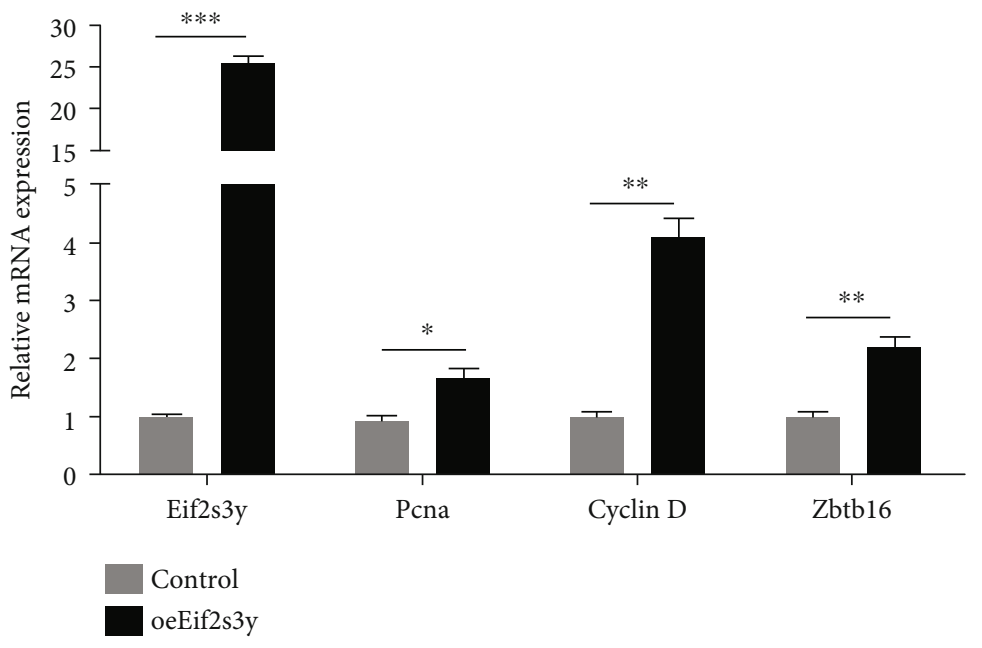

(g)

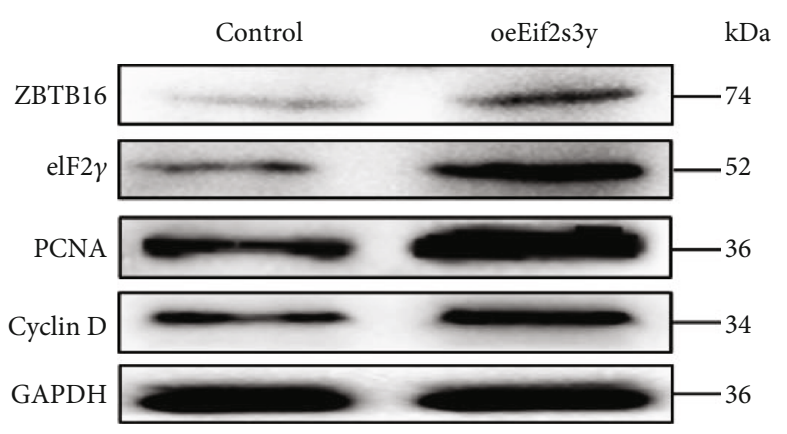

(h)

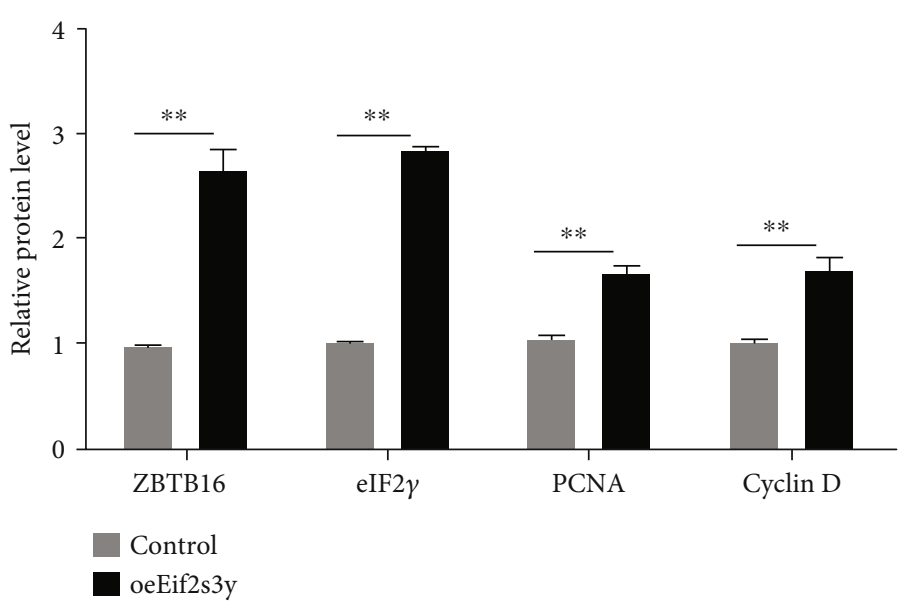

(i)

FIGURE 3: Overexpression of Eif2s3y promotes the proliferation of dairy goat SSCs. (a, b) The schematic of lentivirus plasmid pCDH-CMVMCS-EF1-puro and pCDH-CMV-Eif2s3y-EF1-puro. (c) Typical images of SSCs transfected with Control-Vector (left) or oeEif2s3y-Vector (right). Scale bar, $200 \mu \mathrm{m}$. (d) Proliferation curve of Control and oeEif2s3y SSCs and the results of population doubling time (PDT) determination. (e) EdU incorporation assay of Control (up) and oeEif2s3y (down) SSCs. Scale bar, $400 \mu \mathrm{m}$. (f) The ratio of EdU-positive cells to total cells. The proportion of positive cells is positively correlated with the cell proliferation rate. (g) RT-PCR analysis of the expression levels of Eif2s3y, Pcna, Cyclin D, and Zbtb16 in dairy goat SSCs transfected with Control-Vector or oeEif2s3y-Vector. (h) Western blotting detected the protein expression of ZBTB16, eIF2 $\gamma$, PCNA, and Cyclin D in Control and oeEif2s3y SSCs. GAPDH was used as a loading control. (i) Gray intensity analysis of WB results normalized to GAPDH in (h). Data are presented as mean \pm SD and are represented by three independent repetitions; ${ }^{*} P<0.05,{ }^{* *} P<0.01$, and ${ }^{* * *} P<0.001$.

3.3. Overexpression of Eif2s3y Promotes the Proliferation of Dairy Goat SSCs. The Eif2s3y fragment was inserted into the $\mathrm{pCDH}-\mathrm{CMV}$-MCS-EF1-puro vector, and a recombinant plasmid pCDH-CMV-Eif2s3y-EF1-puro was successfully constructed (Figures 3(a) and 3(b)). The pCDH-Eif2s3y and pCDH lentivirus were collected as described in Materials and Methods. The morphology and function of primary SSCs that we used to verify the direct effects of Eif $2 s 3 y$ have been verified in the past experimental studies $[19,21]$. The primary cells and pure spermatogonia are shown in Supplemental Figure 1A. We examined the expression of several marker genes of SSCs by qRT-PCR and IF staining. The expression of SSC marker genes Zbtb16, GFRa1, and Stra 8 was significantly higher in the pure spermatogonia (Supplemental Figure 1B). The same conclusion was obtained by immunofluorescence staining (Supplemental Figure 1C). We successfully enriched SSCs.

The SSCs were infected with lentivirus pCDH-Eif2s3y or pCDH. After screening by $500 \mathrm{ng} / \mathrm{mL}$ puromycin (Sigma) for one week, oeEif2s3y cells and control cells were established (Figure 3(c)). Interestingly, the morphology of oeEif $2 s 3 y$ cells changed and the edge of colonies became unsmooth, showing a certain extent of differentiation. According to a previous report, the Eif2s $3 y$ defect would block the production of spermatogonia and result in infertility in mice [13]. Our results showed that Eif2s3y promoted the proliferation of goat SSCs, 


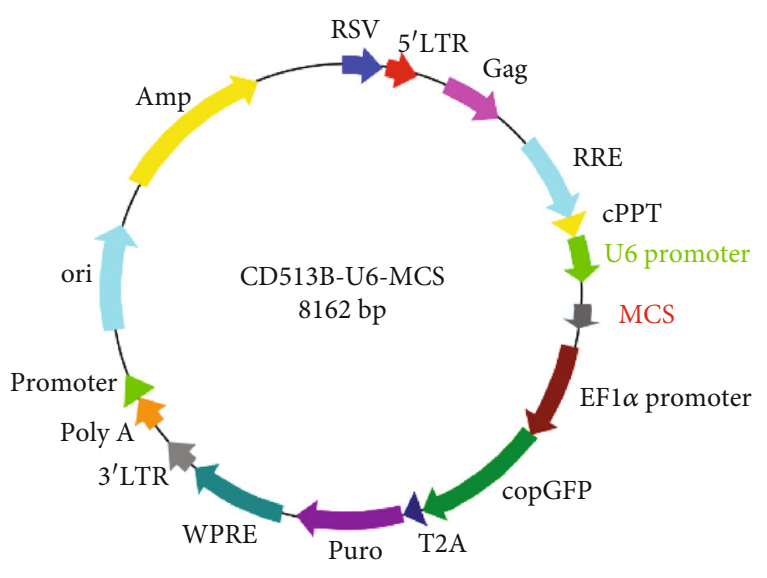

(a)
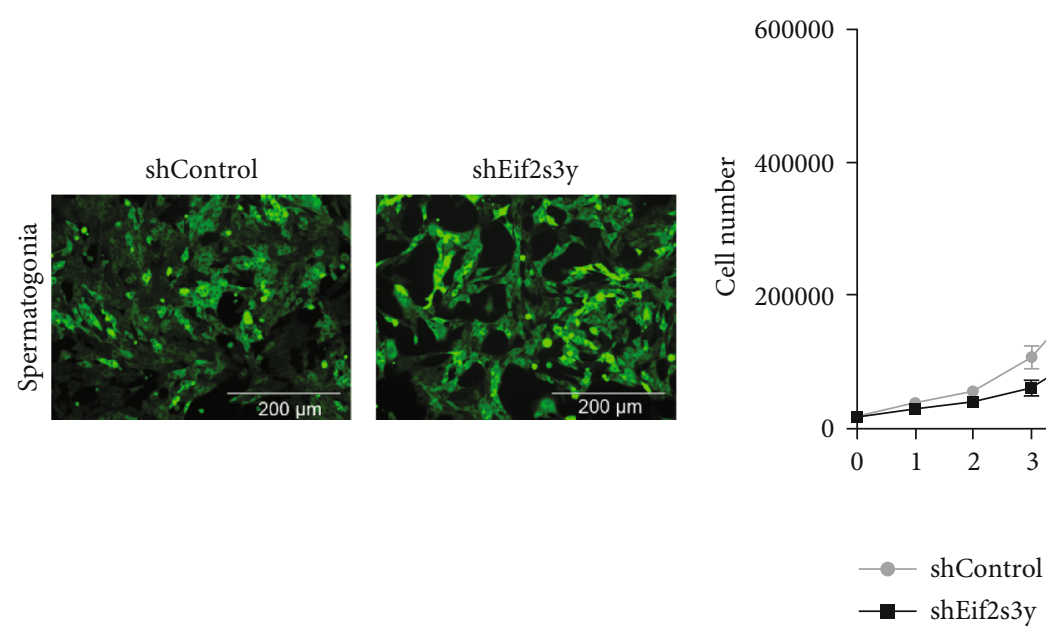

(c)
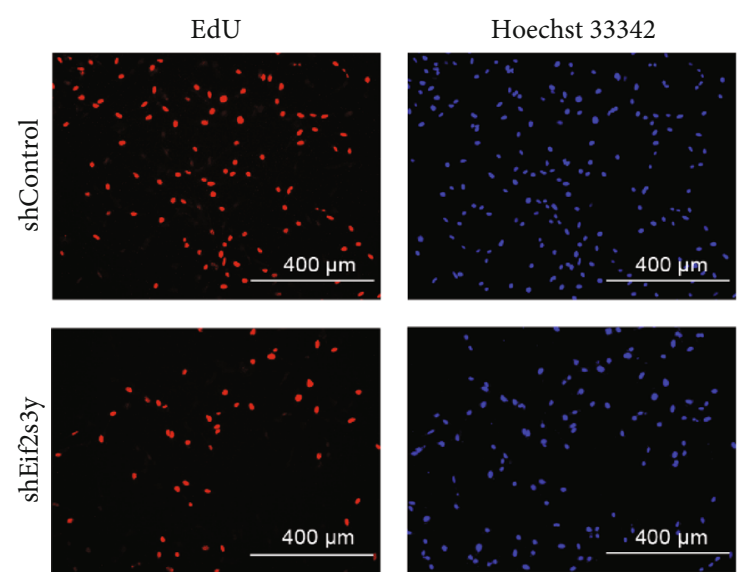

(e)

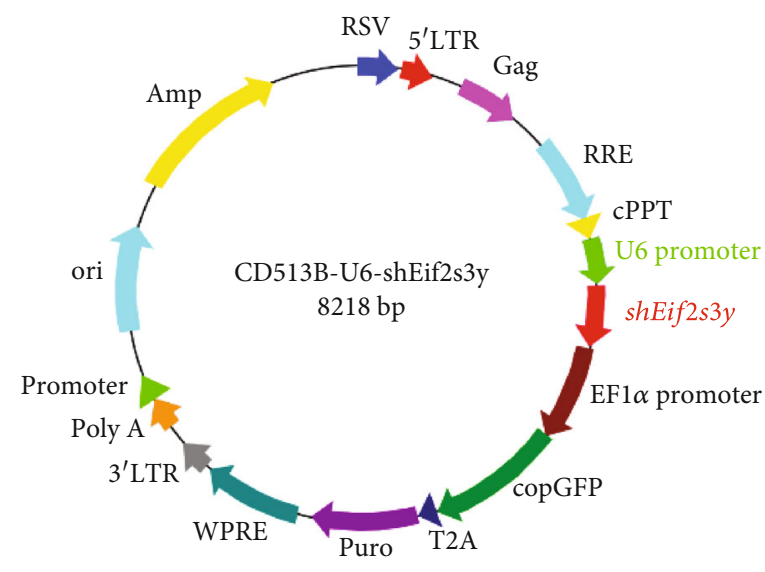

(b)

PDT $($ shControl $)=35.9 \mathrm{~h}$ PDT $($ shEif2s $3 \mathrm{y})=44.8 \mathrm{~h}$

(d)
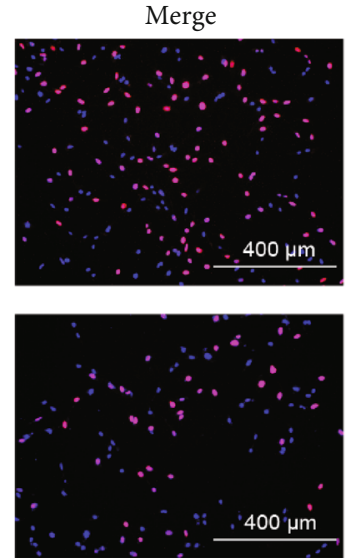

.

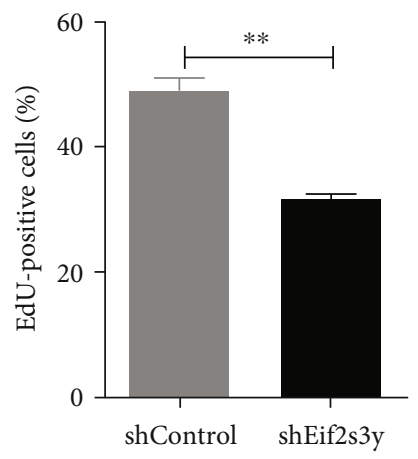

(f)

Figure 4: Continued. 


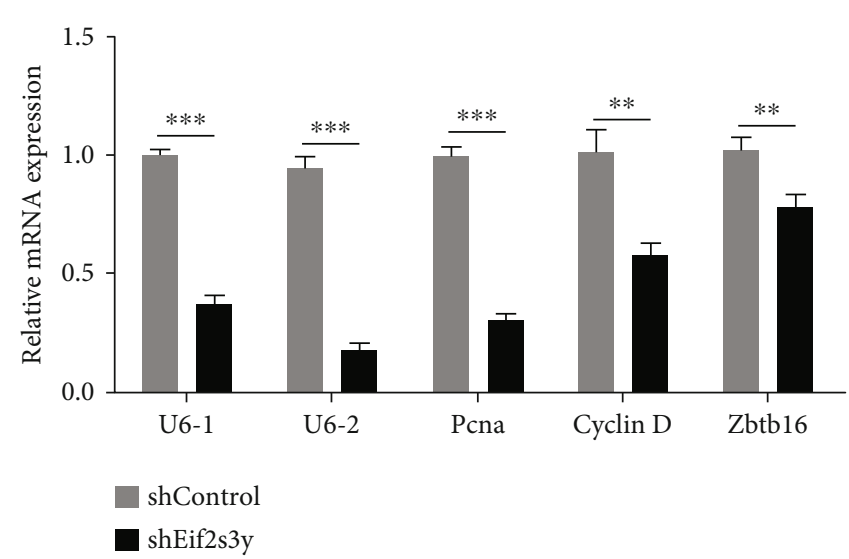

(g)

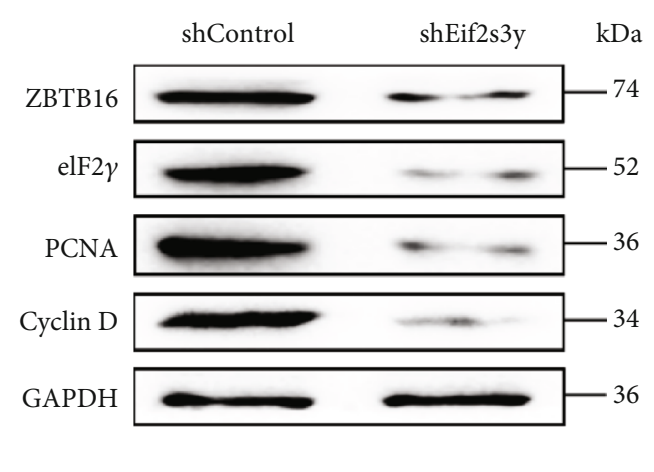

(h)

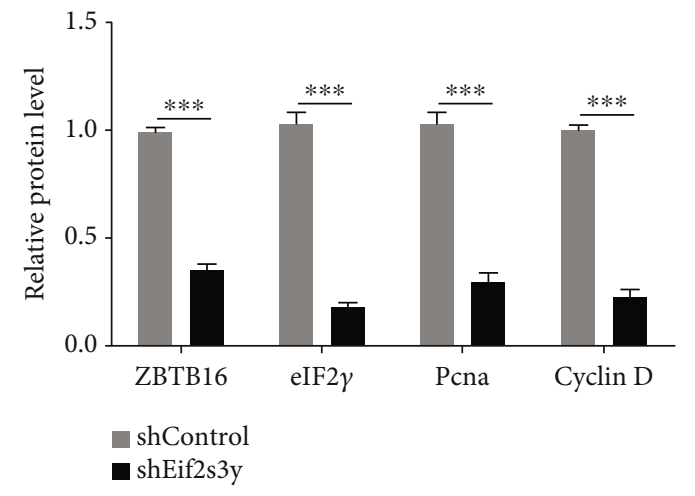

(i)

FIGURE 4: Depletion of Eif2s3y resulted in proliferation abnormality in goat SSCs. (a, b) The schematic of lentivirus plasmid CD513B-U6-MCS and CD513B-U6-shEif2s3y. (c) Typical images of shControl (left) and shEif2s3y (right) SSCs. Scale bar, $200 \mu \mathrm{m}$. (d) Proliferation curve of shControl and shEif2s3y SSCs and the results of population doubling time (PDT) determination. (e) EdU incorporation assay of shControl (up) and shEif2s3y (down) SSCs. Scale bar, $400 \mu \mathrm{m}$. (f) The ratio of EdU-positive cells to total cells. Data are presented as mean \pm SD and are represented by three independent repetitions. (g) RT-PCR analysis of the expression levels of Eif2s3y, Pcna, Cyclin D, and Zbtb16 in shControl and shEif2s3y SSCs in vitro. (h) Western blotting detected the protein expression of ZBTB16, eIF2 $\gamma$, PCNA, and Cyclin D in shControl and shEif2s3y SSCs. GAPDH was used as a loading control. (i) Gray intensity analysis of WB results normalized to GAPDH in (h). Data are presented as mean $\pm \mathrm{SD}$ and are represented by three independent repetitions; ${ }^{*} P<0.05,{ }^{* *} P<0.01$, and ${ }^{* * *} P<0.001$.

as reflected by the higher proliferation rate of oeEif2s3y cells in cell number counting (Figure 3(d)). The population doubling time (PDT) of oeEif2s3y cells was significantly reduced from 34.6 hours to 28.9 hours. The results were further strengthened by EdU incorporation assay (Figures 3(e) and $3(\mathrm{f})$ ). In accordance with these findings, we found that the expression levels of proliferation-associated genes (Pcna, Cyclin D) and self-renewal-associated gene (Zbtb16) were increased in oeEif2s3y cells (Figure $3(\mathrm{~g})$ ). The proportion of red positive cells was consistent with the cell proliferation rate. Western blotting was applied to exam the effect of transgenic Eif2s3y (Figure 3(h)). In oeEif2s3y SSCs, statistical analysis showed that the expression of ZBTB16, eIF2 $\gamma$, Pcna, Cyclin D was higher than in Control SSCs (Figure 3(i)). Collectively, these data demonstrated that overexpression of Eif2s3y promoted the proliferation of goat SSCs.

\subsection{Eif2s3y Deficiency Reversed the Goat SSC Growth Rate.} Since overexpression of Eif2s $3 y$ could promote SSC proliferation, we wondered whether knockdown of Eif2s3y expres- sion would inhibit this proliferation. Recombinant plasmid CD513B-U6-shEif2s3y was successfully constructed (Figures 4(a) and 4(b)). Seven days after infection with lentivirus, RT-PCR analysis confirmed the successful knocking down of Eif2s3y expression (shEif2s3y) in goat SSCs. The efficiency of the two interfering fragments was $60 \%$ or $90 \%$, respectively (Figure $4(\mathrm{~g})$ ). We chose the more efficient U6Vector2 for future experiments (Figures 4(c) and 4(e)).

The population doubling time of shControl and shEif2s3y SSCs was 35.9 or 44.8 hours, respectively (Figure 4(d)). Then, we evaluated the proliferation rate by EdU staining; the percentage of EdU-positive shEif2s3y cells was lower than that of shControl (Figures 4(e) and 4(f)). Compared with the shControl group, the expression levels of proliferation-associated genes Pcna and Cyclin $\mathrm{D}$ and self-renewal-associated gene Zbtb16 in the shEif2s3y group were significantly decreased (Figures $4(\mathrm{~g})$ and $4(\mathrm{~h})$ ). Western blotting analysis got the same results (Figure 4(i)). These experiments showed that Eif2s3y deficiency reversed goat SSC proliferation. 

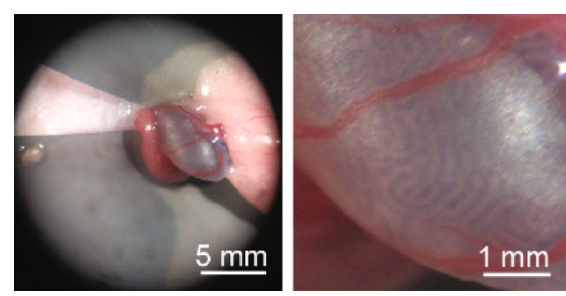

(a)

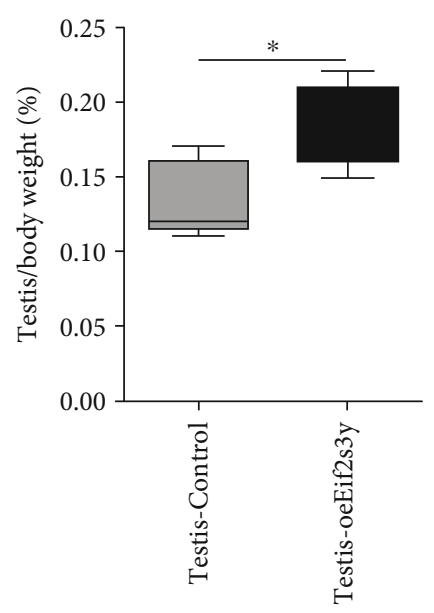

(c)
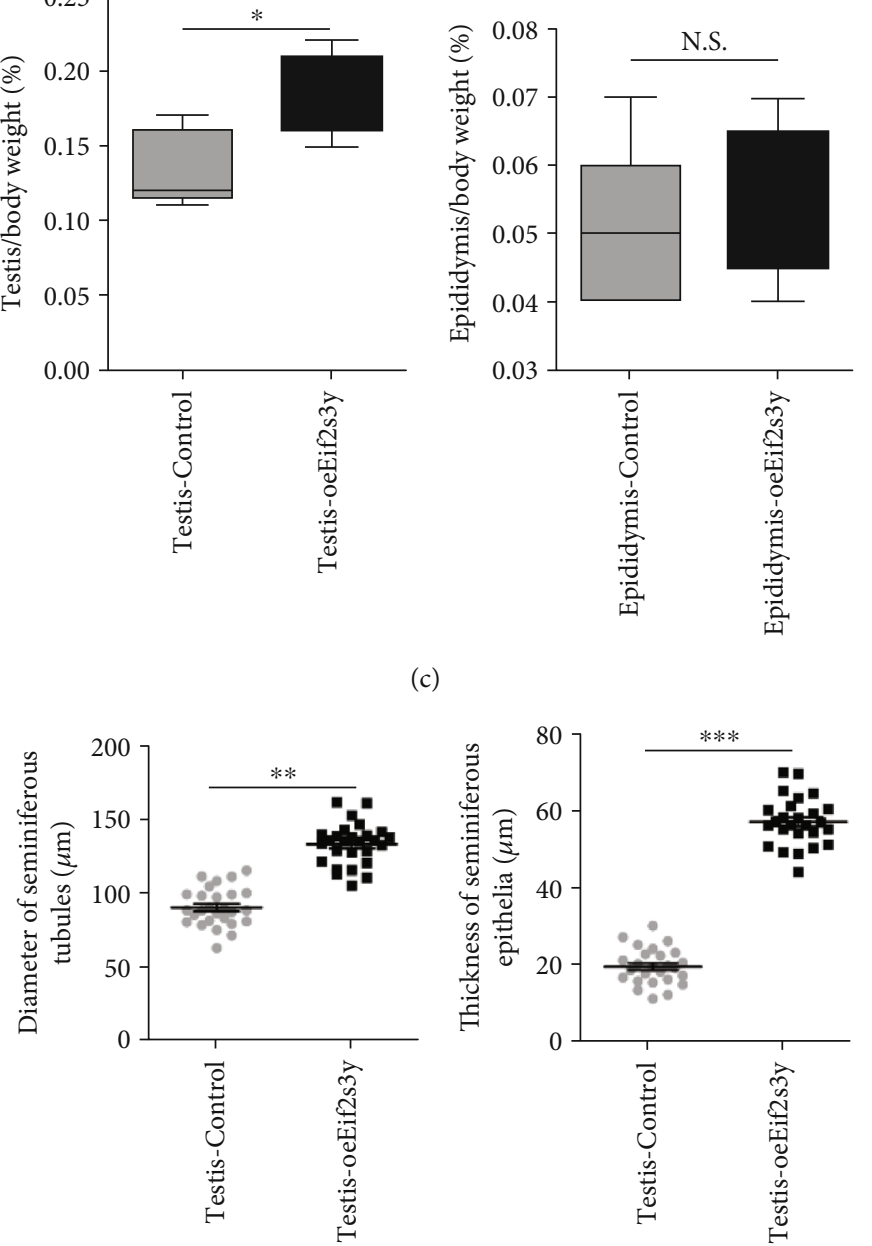

(e)
oeEif2s3y SSCT

Control SSCT

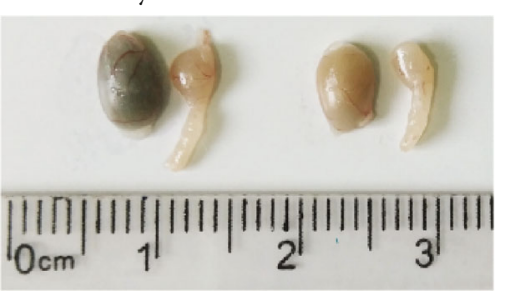

(b)
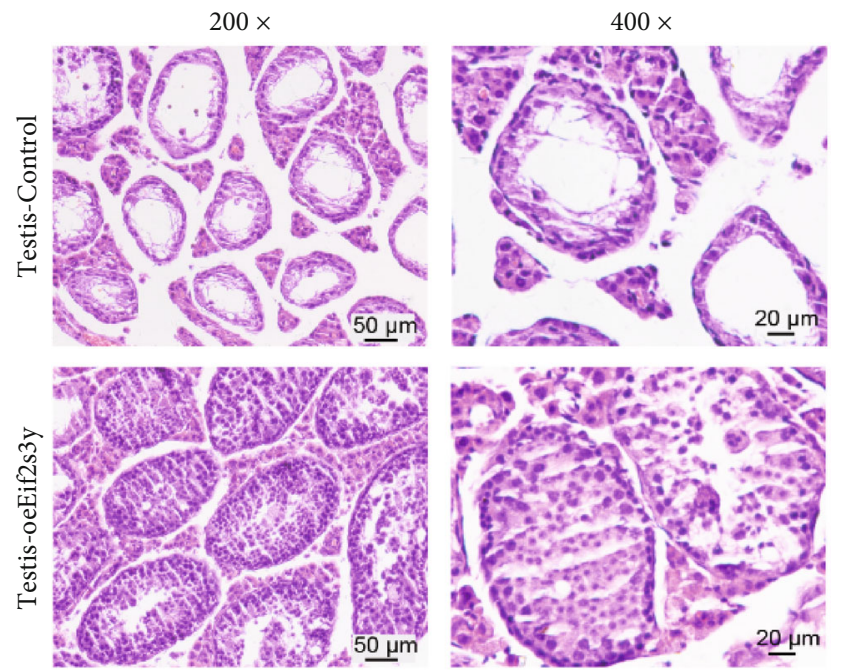

(d)

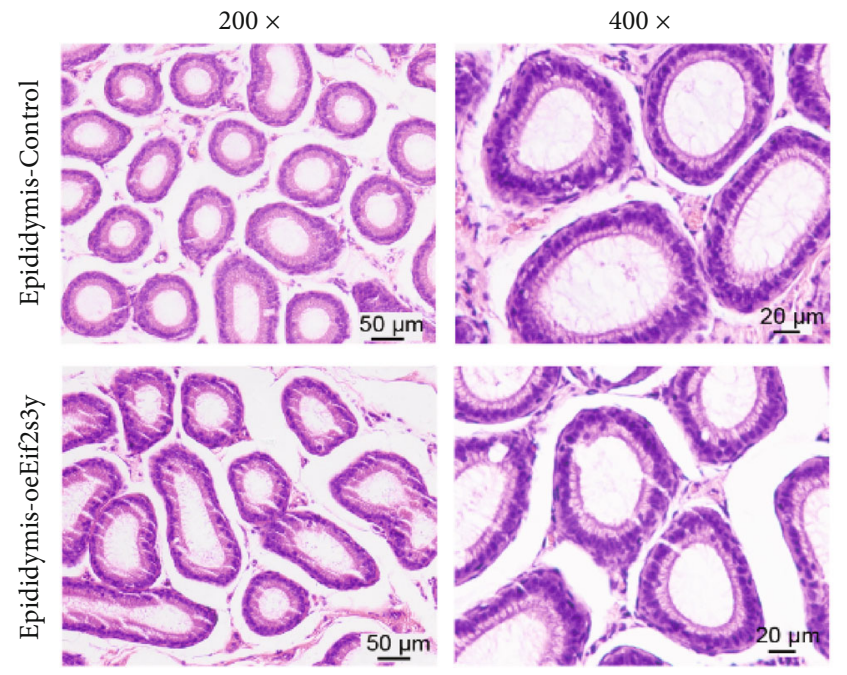

(f)

Figure 5: Continued. 


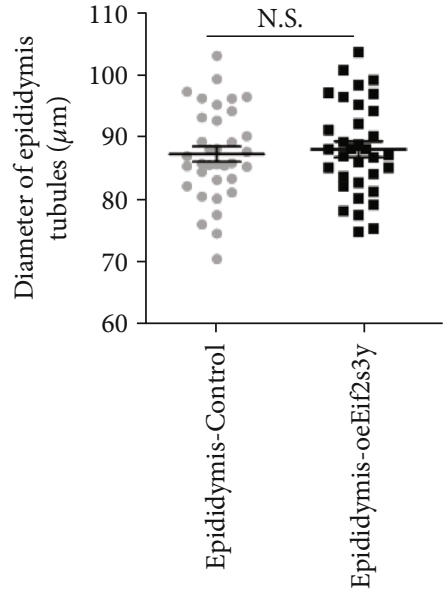

(g)
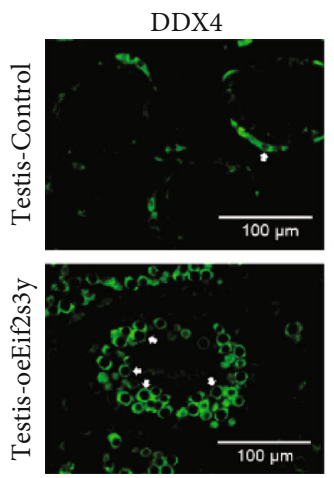

ZBTB16
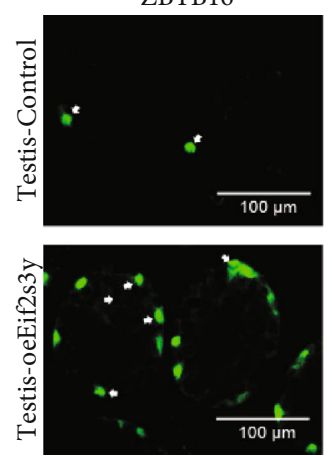

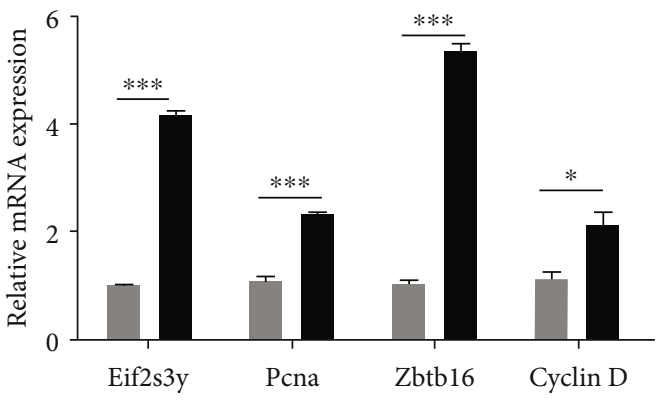

Testis-Control

Testis-oeEif2s3y (h)
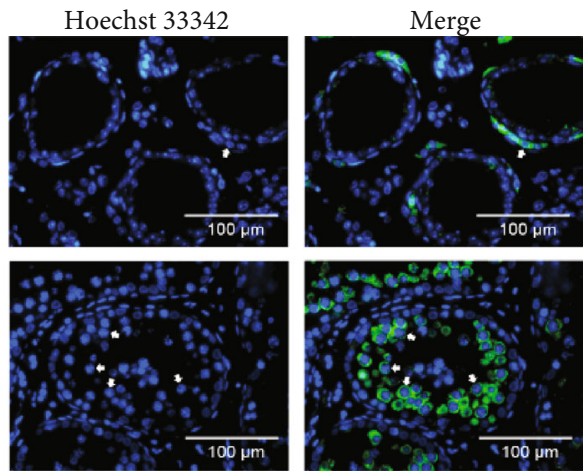

(i)

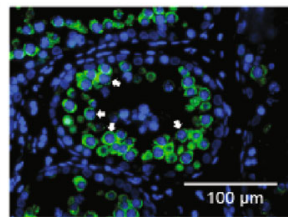

Hoechst 33342
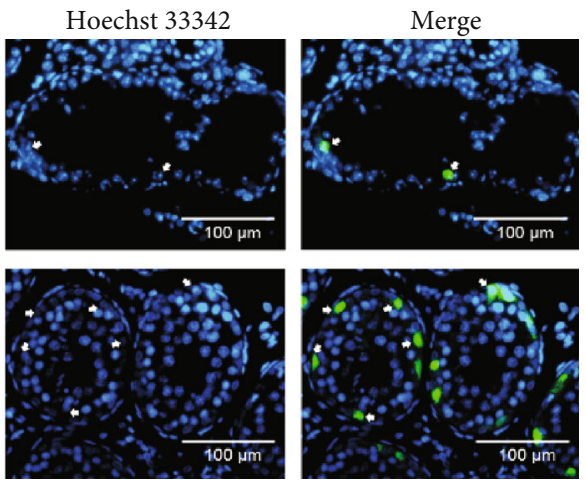

(j)

Figure 5: Eif2s3y promotes the colonization of goat SSCs in SSCT. (a) OeEif2s3y or Control SSCs were injected into the seminiferous tubules of busulfan-treated mice. The figure here shows two typical pictures of the testis during injection. Scale bars, $1 \mathrm{~mm}$ and $5 \mathrm{~mm}$ as indicated. (b) Morphology of testes and epididymides transfected with oeEif2s3y or Control SSCs. (c) The testicular (left) and epididymis (right) weight/body mass ratio in two groups. (d) H\&E staining of the mouse testis 30 days after injection. Scale bars, $50 \mu \mathrm{m}$ (left) and $20 \mu \mathrm{m}$ (right). (e) Statistical plots of the diameter of seminiferous tubules (left) and the thickness of seminiferous epithelia (right) from oeEif2s3y and Control SSC-transplanted mice. Each group counted at least 30 round seminiferous tubules from 10 mice. (f) H\&E staining of the mouse epididymis 30 days after injection. Scale bars, $50 \mu \mathrm{m}$ (left) and $20 \mu \mathrm{m}$ (right). (g) Statistical plots of the diameter of epididymis tubules from oeEif2s $3 y$ and Control SSC-transplanted mice. Each group counted at least 30 epididymis tubules from 10 mice. (h) RT-PCR analysis of the expression of Eif2s3y, Pcna, Zbtb16, and Cyclin D in Testis-Control and Testis-oeEif2s3y. (i) Immunofluorescence staining of DDX4 (green) in SSC-transplanted testes. The nuclei were stained with Hoechst 33342 (blue). Scale bar, $100 \mu \mathrm{m}$. These white arrows represented typical DDX4-positive germ cells. DDX4 is a representative marker for pan-germ cells. (j) Immunofluorescence staining of ZBTB16 (green) in SSC-transplanted testes. The nuclei were stained with Hoechst 33342 (blue). Scale bar, $100 \mu \mathrm{m}$. These white arrows represented typical ZBTB16-positive spermatogonial stem cells. ZBTB16 is a representative marker for undifferentiated spermatogonia. Data are presented as mean $\pm \mathrm{SD}$ and are represented by three independent repetitions; ${ }^{*} P<0.05,{ }^{* *} P<0.01$, and ${ }^{* * *} P<0.001 ; \mathrm{N} . \mathrm{S}$. means $P \geq 0.05$. 


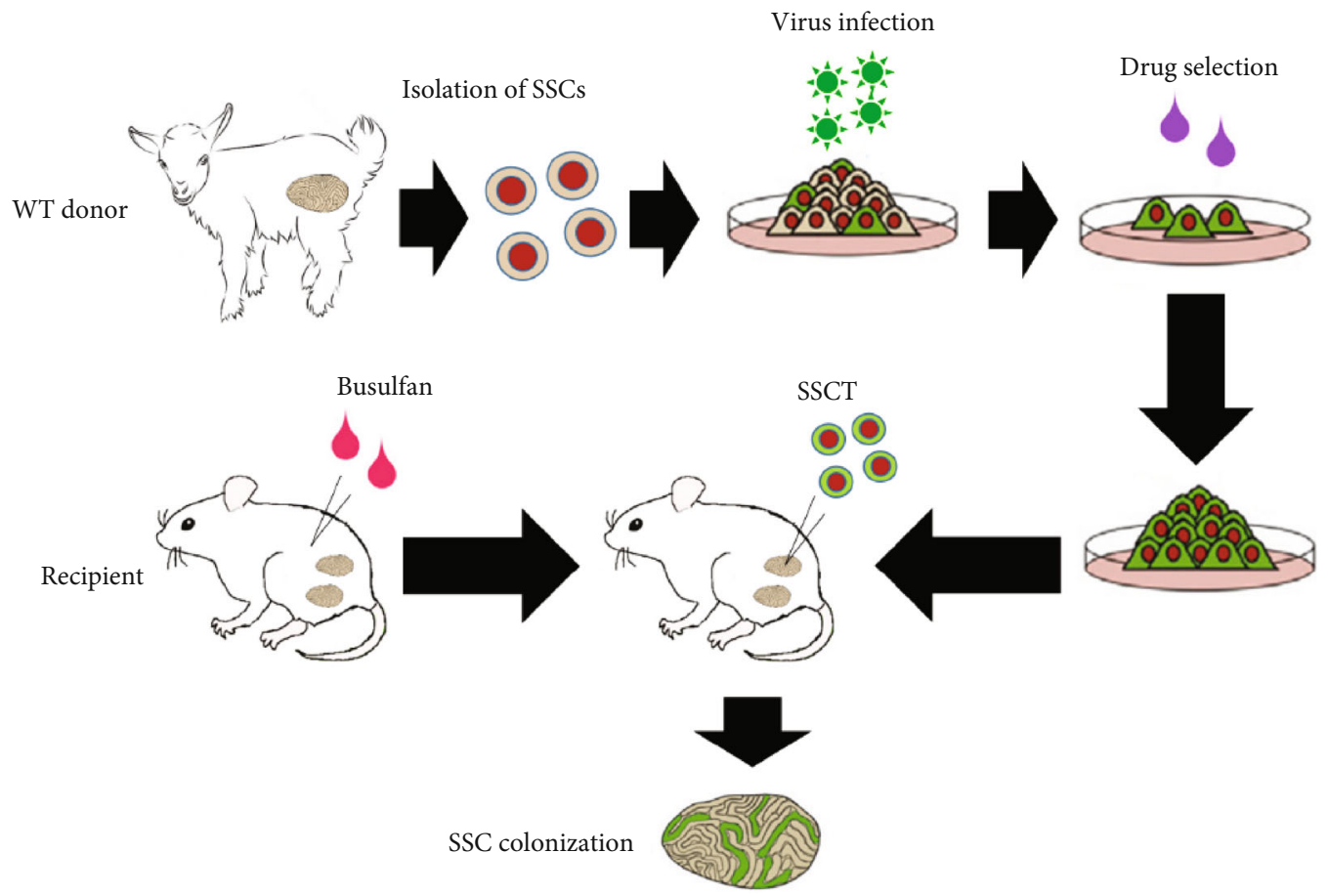

(a)

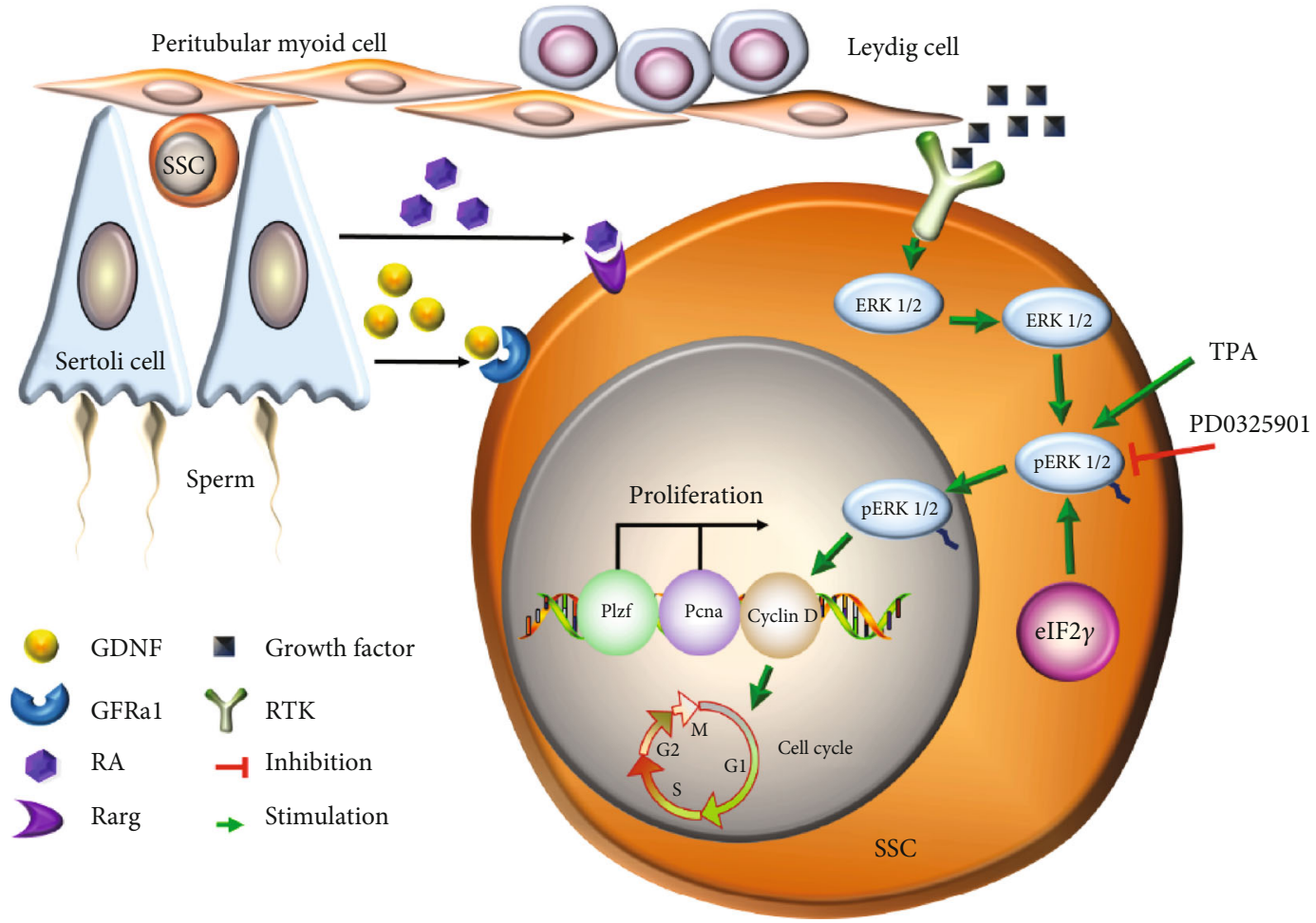

(b)

Figure 6: A schematic diagram illustrating Eif2s3y functions in goat SSCs. (a) Schematic diagram of seminiferous tubule transplantation. (b) A proposed model for Eif2s3y activating the downstream ERK signaling pathway to regulate proliferation genes in goat SSCs. This finding may provide an important basis for future works regarding male spermatogenic cell restoration in large animals.

3.5. Eif2s3y Could Increase the Colonization Rate of Goat SSCs in SSCT. Spermatogonial stem cell transplantation technology has been an effective method to study SSCs since 1994 $[1,26,33]$. Some previous research had proved the reliability of our transplantation technique [17]. To investigate the contribution of Eif2s $3 y$ in SSCs, oeEif2s3y SSCs and Control SSCs were transferred into the seminiferous tubules of twenty infertile mice treated with busulfan (Figures 5(a) and 6(a)). 
Control+DMSO

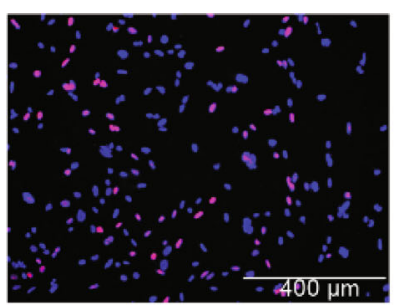

oeEif2s3y+DMSO

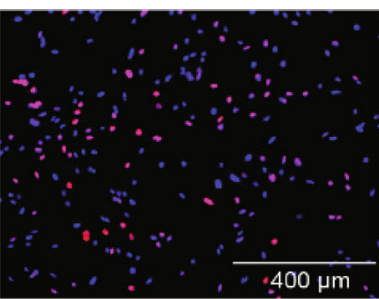

Control+PD0325901

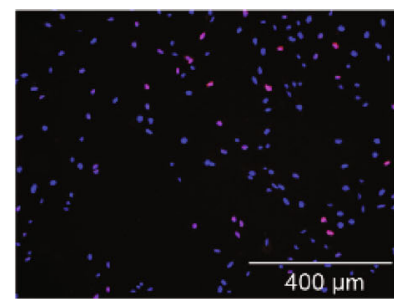

(a)
oeEif2s3y+PD0325901

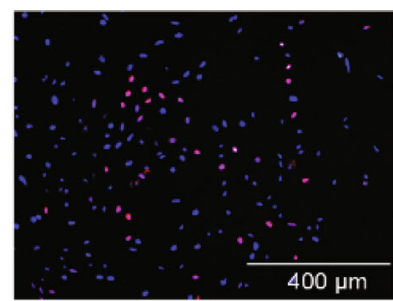

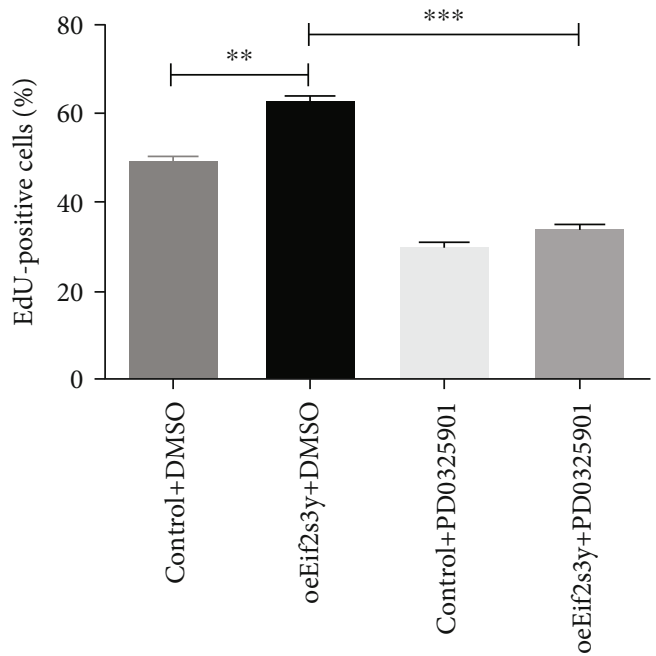

(b)

shEif2s3y+DMSO

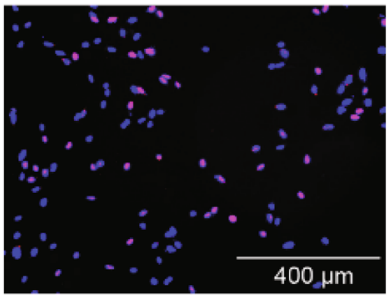

shControl+TPA

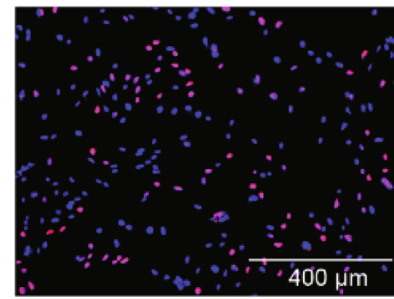

shEif2s3y+TPA

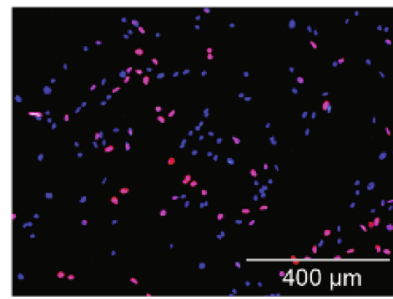

(c)

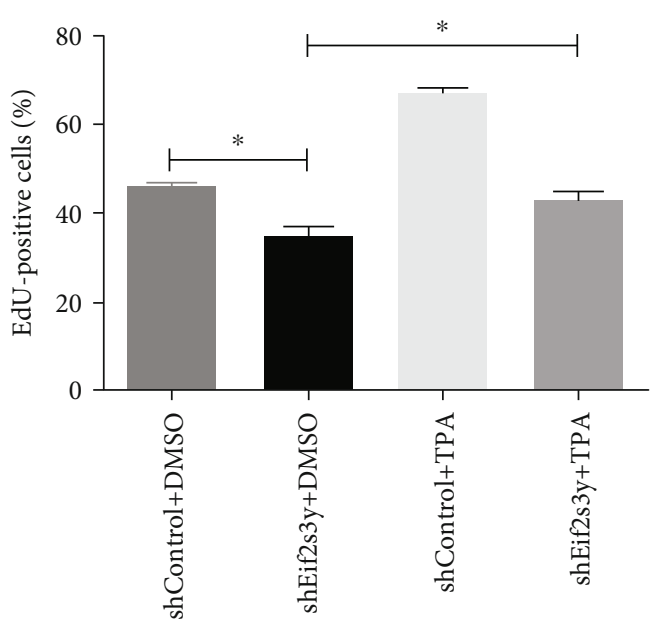

(d)

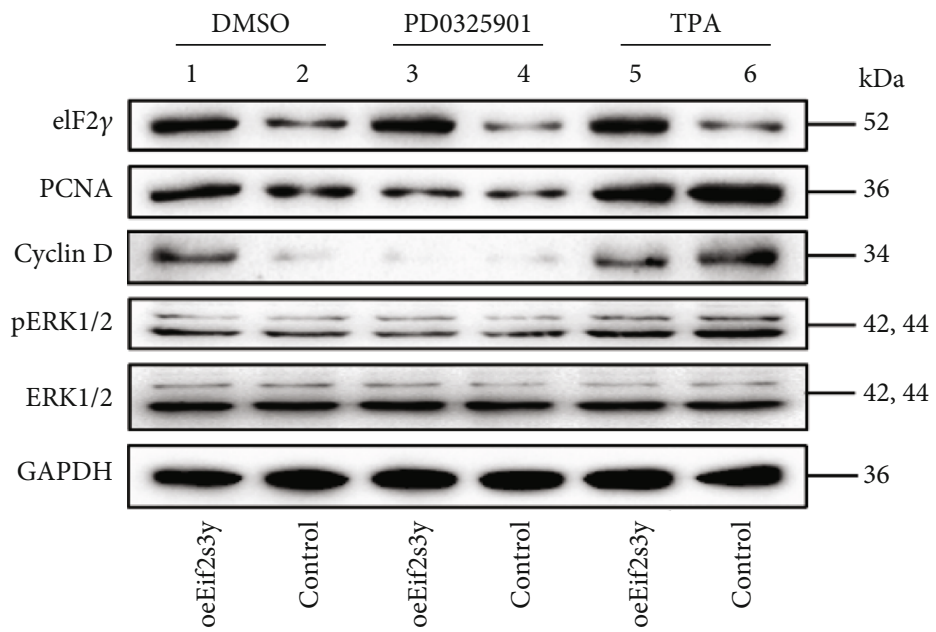

(e)

Figure 7: Continued. 


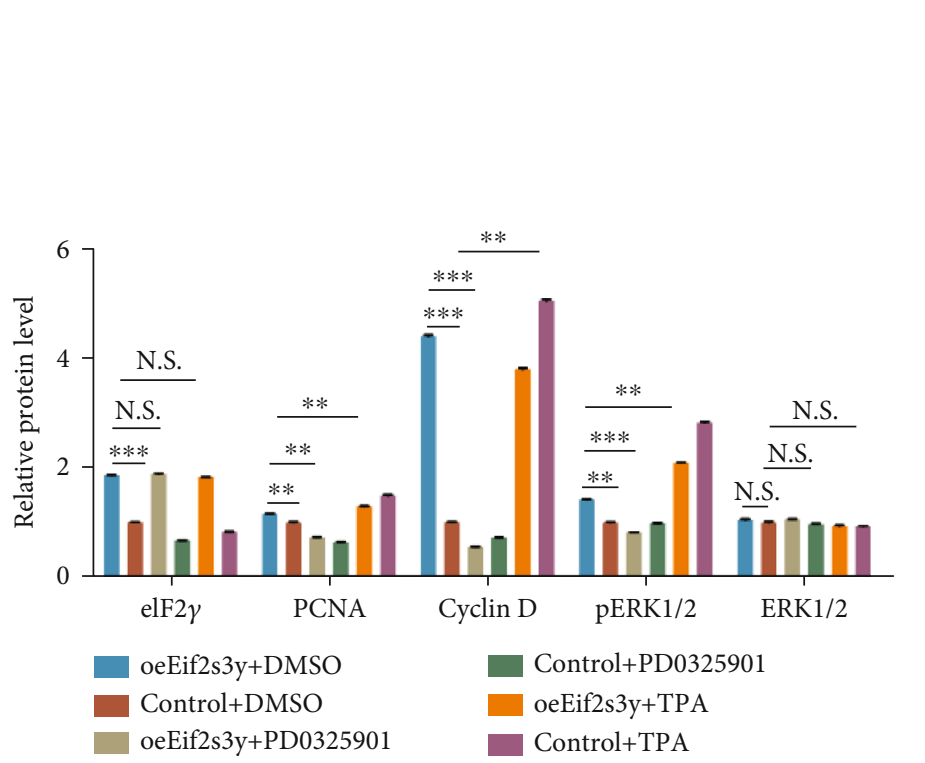

(f)

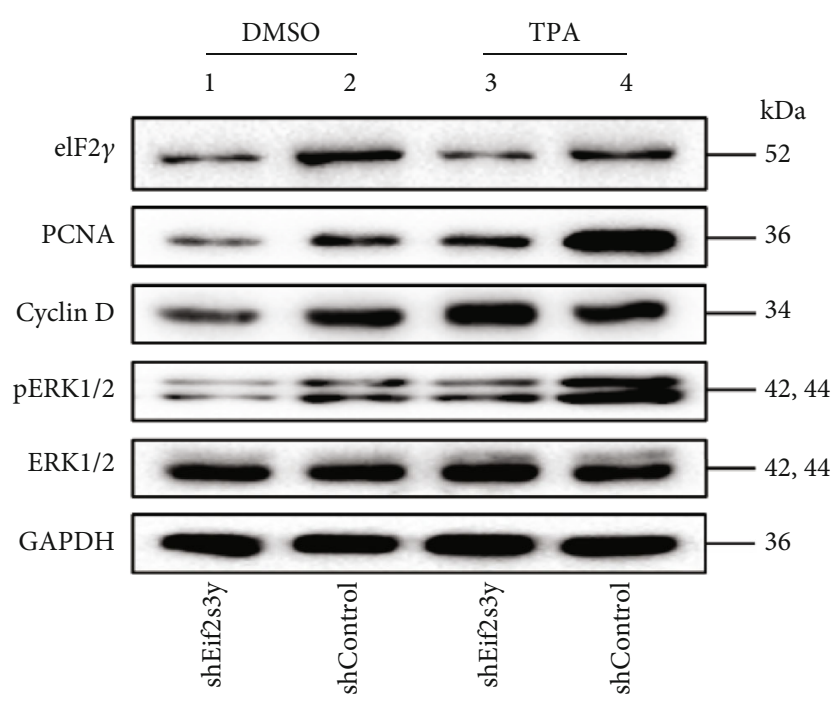

(g)

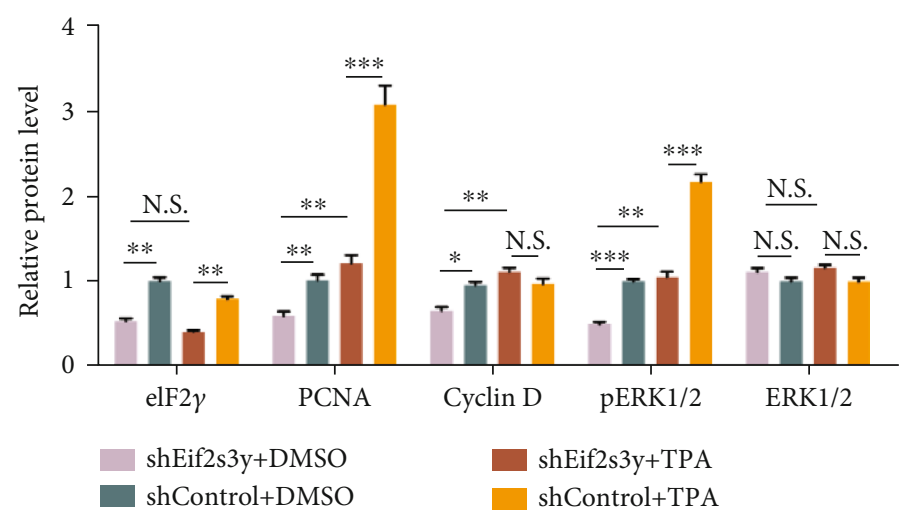

(h)

FIGURE 7: Eif2s3y promotes SSC proliferation by activating the ERK signaling pathway. Control and oeEif2s3y SSCs were treated with $1 \mu \mathrm{M}$ ERK pathway inhibitor PD0325901 or $10 \mu \mathrm{M}$ ERK pathway activator TPA for $24 \mathrm{~h}$, respectively. shControl and shEif2s3y SSCs were treated with $10 \mu \mathrm{M}$ ERK pathway activator TPA for $24 \mathrm{~h}$. DMSO was used for the control group. (a) EdU incorporation assay of Control and oeEif2s3y SSCs added with ERK pathway inhibitor PD0325901 or DMSO. Scale bar, $400 \mu \mathrm{m}$. (b) The percentage of EdU-positive cells to total cells. The proportion of positive cells is positively correlated with the cell proliferation rate. (c) EdU incorporation assay of shControl and shEif2s3y SSCs added with ERK pathway activator TPA or DMSO. Scale bar, $400 \mu \mathrm{m}$. (d) The percentage of EdU-positive cells to total cells. (e) Western blotting detected the protein expression of eIF2 $\gamma$, PCNA, Cyclin D, pERK1/2, and ERK1/2 in SSCs treated as indicated. GAPDH was used as a loading control. (f) Gray intensity analysis of WB results normalized to GAPDH in (e). (g) Western blotting detected the protein expression of eIF2 $\gamma$, PCNA, Cyclin D, pERK1/2, and ERK1/2 in SSCs treated as indicated. (h) Gray intensity analysis of WB results normalized to GAPDH in $(\mathrm{g})$. Data are presented as mean $\pm \mathrm{SD}$ and are represented by three independent repetitions; ${ }^{*} P<0.05,{ }^{* *} P<0.01$, and ${ }^{* * *} P<0.001 ;$ N.S. means $P \geq 0.05$.

Testes transplanted with oeEif2s3y SSCs were heavier than those in the control group $(P=0.014)$, while the weight of the epididymis did not change significantly $(P=0.43)$ (Figures $5(\mathrm{~b})$ and $5(\mathrm{c}))$.

H\&E staining of the transplanted testes showed that more germ cells were observed in the Eif2s3y group (Figure 5(d)). Additionally, the diameter of seminiferous tubules $(P<0.01)$ and the thickness $(P<0.001)$ of seminiferous epithelia were significantly increased in the Eif2s3y group (Figure 5(e)). However, the diameter of epididymis tubules was not significant between these two groups (Figures 5(f) and $5(\mathrm{~g})$ ). In addition, RT-PCR analysis showed that Eif2s $3 y$, Pcna, Zbtb16, and Cyclin D were overexpressed in the
oeEif2s3y group (Figure 5(h)). Importantly, immunofluorescence staining showed that the Eif2s3y group had more DDX4-positive germ cells and more ZBTB16-positive SSCs in the testes (Figures 5(i) and 5(j)). Thus, overexpression of Eif2s3y might contribute to improving the survival rate and proliferation of goat SSCs in SSCT. However, no sperm was found in either group, which might be caused by different species (Figure 5(d)).

3.6. Eif2s3y Promotes the Proliferation of SSCs by Activating the ERK Signaling Pathway. To confirm the proliferation mechanism of Eif2s3y in goat SSCs, we investigated the proliferation-related signaling pathways through western 
blotting in oeEif2s3y and shEif2s3y SSCs. Our previous study found that the expression of Eif2s $3 y$ increased the phosphorylation level of ERK. Then, SSCs were treated by $1 \mu \mathrm{M}$ ERK pathway inhibitor PD0325901 [31] or $10 \mu \mathrm{M}$ ERK pathway activator TPA [32] for 24 hours, respectively. DMSO was used for the control group. As expected, PD0325901 treatment significantly inhibited the proliferation of oeEif2s $3 y$ SSCs, while TPA treatment efficiently restored the proliferation of SSCs transplanted with shEif2s $3 y$, as analyzed by EdU incorporation assay (Figures 7(a)-7(d)).

Western blotting analysis was further conducted to explore the underlying mechanisms, and we found that the MEK-ERK signal could directly and indirectly participate in the proliferation of SSCs. When ERK signaling was activated by Eif2s3y overexpression or by TPA treatment, the expression levels of PCNA and Cyclin D were both increased. The results showed that the ERK signaling blockade, either by Eif2s3y interference or by PD0325901 treatment, significantly inhibited the expression levels of PCNA and Cyclin $\mathrm{D}$ (Figures 7(e) and 7(f)). In comparison, the results showed that the ERK signaling blockaded, either by Eif2s $3 y$ interference or by PD0325901 treatment, significantly inhibited the expression levels of PCNA and Cyclin D, both of which were essentially needed during cell proliferation (Figures $7(\mathrm{~g})$ and $7(\mathrm{~h})$ ). According to the above experimental results, we hypothesized that Eif2s3y activated the downstream ERK signaling pathway to regulate proliferation genes.

According to the above experimental results, we proposed a mechanism diagram of how Eif2s3y activated the downstream ERK signaling pathway to promote SSC proliferation and restoration of male spermatogenic cells in goat testes (Figure 6(b)).

\section{Discussion}

Eif2s $3 y$ is widely expressed in different male animals and recognized as a translation initiation factor $[11,34]$. In recent studies, we found that Eif2s3y could regulate the proliferation of goat SSCs. The expression levels of proliferation- and selfrenewal-related genes Cyclin D, Cyclin A, Pcna, and Plzf were upregulated in oeEif2s3y SSCs and downregulated in shEif2s3y SSCs (Figures 3(g) and 4(g)). Our results showed that Eif2s $3 y$ played an important role in male reproduction of dairy goats, and these results were consistent with previous studies in mice that Eif2s $3 y$ played a critical role in male spermatogenesis $[14,15,35]$. Importantly, we found a regulatory pathway of Eif2s $3 y$ in male reproduction with ERK signaling involved. This finding was in line with previous studies reported by us and other groups that the MEK/ERK signaling pathway played an important role in cell proliferation, differentiation, and cell cycle progression [36, 37]. However, how Eif2s3y regulates ERK signaling remains to be further studied.

SSCT technology is an effective method to identify the characteristics of SSCs cultured in vitro [1]. We transferred oeEif2s $3 y$ SSCs into the seminiferous tubules of infertile mice and found that Eif2s3y could enhance the colonization of germ cells (Figure 5(d)). Moreover, no mature sperm was observed in the epididymis (Figure 5(e)). The blood-testis barrier (BTB) made goat SSCs transplanted into mouse testes survive [5]. However, the species relationship between goats and mice was so far that mice could not produce goat sperms. As a contrast, we injected CD513B-U6-shEif2s3y lentivirus into the seminiferous tubules of wild-type mice and found that spermatogenesis was blocked and the germ cells in seminiferous tubules were very loose. Another research group directly knocked out the mouse Eif2s $3 y$ gene with TALEN technology, which led to testicular hypoplasia and male infertility [13]. More generally, all these results of animal experiments in vivo indicated that Eif2s3y played an important role in spermatogenesis.

Our bioinformatics analysis showed that Eif2s3y was highly conserved among different species (Figure 2(c)). Therefore, the interference experiment of mice might also be applicable to goats. Dairy goat Eif2s3y was located on the Y chromosome and encoded a 471 amino acid protein which contained a compact zinc finger domain and an $\mathrm{N}$-terminal GTP binding domain (Figures 2(d) and 2(e)). In mice, Eif2s3x was a homologous gene of Eif2s3y and shared 98\% of amino acid sequence identity and almost all of the RNA binding domains with Eif2s3y [35]. However, recent studies have found that these two genes might not completely replace each other. Eif2s3x has been found to play an irreplaceable role in the early development of organs such as the brain and pancreas [38-40]. Meanwhile, a recent study showed that Eif $2 s 3 y$ was more effective in masculinizing mice during sex growth at 12.5 days of mouse embryonic development [41]. However, in dairy goats, it was still unclear whether a homologous gene of Eif2s3y exists and how they worked together. Thus, future work is essentially needed to answer these questions.

In conclusion, our study found a novel role of Eif2s $3 y$ in the male reproduction of dairy goats. This finding might provide an important basis for the repair of male infertility and spermatogonial stem cell transplantation toward realizing the regeneration of germplasm in large animals.

\section{Data Availability}

The data that support the findings of this study are available from the corresponding author upon reasonable request.

\section{Conflicts of Interest}

The authors declare that no conflict of interest exists.

\section{Authors' Contributions}

The contributions of the authors involved in this study are as follows: JLH: experimental design and financial support; MFZ, NL, and WQL: performance of cellular and molecular experiments, data analysis, and manuscript preparation; $\mathrm{XMD}, \mathrm{YDW}, \mathrm{DHY}$, and ZZ: preparation of lentivirus injection, mouse model, and primary SSCs; and FLM, NL, SP, SQZ, XH, CLB, and GPL: assistance of experiments and revision and discussion of the manuscript. Mengfei Zhang and $\mathrm{Na} \mathrm{Li}$ contributed equally to this work. The author order was determined by negotiation. 


\section{Acknowledgments}

This work was supported by the National Natural Science Foundation of China (32002246, 31572399, 32072806, and 31272518), the Fundamental Research Funds for the Central Universities (2452020157), and the China National Basic Research Program (JFYS 2016YFA0100203).

\section{Supplementary Materials}

Supplementary 1. Supplemental Figure 1: isolation and enrichment of dairy goat spermatogonia. (A) The primary cells and pure spermatogonia isolated from healthy dairy goat testes; scale bar $=200 \mu \mathrm{m}$. (B) RT-PCR analysis of the expression of Zbtb16, GFRa1, and Stra8 in primary cells and pure spermatogonia. Data are presented as mean $\pm \mathrm{SD}$ and are represented by three independent repetitions; ${ }^{*} P<$ $0.05,{ }^{* *} P<0.01$. (C) Immunofluorescence staining of ZBTB16 (up), STRA8 (middle), and GFRa1 (down) in pure spermatogonia. The nuclei were stained with Hoechst 33342 (blue). Scale bar, $200 \mu \mathrm{m}$. ZBTB16, STRA8, and GFRal are representative markers for SSCs.

Supplementary 2. Supplemental Figure 2: some additional pictures. (A) Immunofluorescence staining of mouse (up) and goat (down) testes. Only IgG $(1: 200)$ was used for the first antibody, and the fluorescent secondary antibody was used normally. The nuclei were stained with Hoechst 33342 (blue). Scale bar, $200 \mu \mathrm{m}$. Species-specific IgG antibody for immunofluorescence experiments as the parallel negative antigen control to prove that there was no false positive in our immunofluorescence staining. (B) Immunofluorescence staining of SOX9 (up) and StAR (down) in goat testes. The nuclei were stained with Hoechst 33342 (blue). Scale bar, $100 \mu \mathrm{m}$. SOX9 is a representative marker for Sertoli cells, and StAR is a representative marker for Leydig cells. (C) $\mathrm{H} \& \mathrm{E}$ staining of 3-month-old and 24-month-old goat testes. Scale bars, $50 \mu \mathrm{m}$ (left) and $20 \mu \mathrm{m}$ (right).

Supplementary 3. Supplemental Figure 3: full unedited images. (A) Full unedited PCR images of Figure 2(a) data. (B) Full unedited western blotting images of Figure 7(e) data. (C) Full unedited western blotting images of Figure 7(g) data.

Supplementary 4. Supplemental Table 1: the sequence and length of primers used in qRT-PCR amplification.

Supplementary 5. Supplemental File 1: normal distribution test for experimental data.

Supplementary 6. Supplemental File 2: experimental information for qRT-PCR.

\section{References}

[1] R. L. Brinster and M. R. Avarbock, "Germline transmission of donor haplotype following spermatogonial transplantation," Proceedings of the National Academy of Sciences of the United States of America, vol. 91, no. 24, pp. 1130311307, 1994.

[2] H. Nishimura and S. W. L'Hernault, "Spermatogenesis," Current Biology, vol. 27, no. 18, pp. R988-R994, 2017.
[3] M. C. Nagano, "Homing efficiency and proliferation kinetics of male germ line stem cells following transplantation in mice," Biology of Reproduction, vol. 69, no. 2, pp. 701-707, 2003.

[4] K. Guan, K. Nayernia, L. S. Maier et al., "Pluripotency of spermatogonial stem cells from adult mouse testis," Nature, vol. 440, no. 7088, pp. 1199-1203, 2006.

[5] M. Kanatsu-Shinohara, N. Ogonuki, S. Matoba, A. Ogura, and T. Shinohara, "Autologous transplantation of spermatogonial stem cells restores fertility in congenitally infertile mice," Proceedings of the National Academy of Sciences of the United States of America, vol. 117, no. 14, pp. 7837-7844, 2020.

[6] A. P. Grigg, R. McLachlan, J. Zajac, and J. Szer, "Reproductive status in long-term bone marrow transplant survivors receiving busulfan-cyclophosphamide $(120 \mathrm{mg} / \mathrm{kg})$," Bone Marrow Transplantation, vol. 26, no. 10, pp. 1089-1095, 2000.

[7] M. Delessard, J. Saulnier, A. Rives, L. Dumont, C. Rondanino, and N. Rives, "Exposure to chemotherapy during childhood or adulthood and consequences on spermatogenesis and male fertility," International Journal of Molecular Sciences, vol. 21, no. 4, p. 1454, 2020.

[8] R. L. Brinster, "Male germline stem cells: from mice to men," Science, vol. 316, no. 5823, pp. 404-405, 2007.

[9] M. Hutka, L. B. Smith, and R. T. Mitchell, "Xenotransplantation as a model for human testicular development," Differentiation, vol. 97, pp. 44-53, 2017.

[10] E. Ntemou, P. Kadam, D. van Saen et al., "Complete spermatogenesis in intratesticular testis tissue xenotransplants from immature non-human primate," Human Reproduction, vol. 34, no. 3, pp. 403-413, 2019.

[11] W. C. Merrick, "Mechanism and regulation of eukaryotic protein synthesis," Microbiological Reviews, vol. 56, no. 2, pp. 291315, 1992.

[12] B. S. Shin, J. R. Kim, S. E. Walker, J. Dong, J. R. Lorsch, and T. E. Dever, "Initiation factor eIF2 $\gamma$ promotes eIF2-GTPMet- $\mathrm{tRNA}_{\mathrm{i}}^{\text {Met }}$ ternary complex binding to the $40 \mathrm{~S}$ ribosome," Nature Structural \& Molecular Biology, vol. 18, no. 11, pp. 1227-1234, 2011.

[13] Y. Matsubara, T. Kato, K. Kashimada et al., "TALEN-mediated gene disruption on Y chromosome reveals critical role of EIF2S3Y in mouse spermatogenesis," Stem Cells and Development, vol. 24, no. 10, pp. 1164-1170, 2015.

[14] S. Mazeyrat, N. Saut, V. Grigoriev et al., "A Y-encoded subunit of the translation initiation factor Eif2 is essential for mouse spermatogenesis," Nature Genetics, vol. 29, no. 1, pp. 49-53, 2001.

[15] Y. Yamauchi, J. M. Riel, Z. Stoytcheva, and M. A. Ward, “Two $\mathrm{Y}$ genes can replace the entire $\mathrm{Y}$ chromosome for assisted reproduction in the mouse," Science, vol. 343, no. 6166, pp. 69-72, 2014.

[16] Y. Yamauchi, J. M. Riel, V. A. Ruthig, E. A. Ortega, M. J. Mitchell, and M. A. Ward, "Two genes substitute for the mouse Y chromosome for spermatogenesis and reproduction," Science, vol. 351, no. 6272, pp. 514-516, 2016.

[17] N. Li, W. Ma, Q. Shen et al., "Reconstitution of male germline cell specification from mouse embryonic stem cells using defined factors in vitro," Cell Death and Differentiation, vol. 26, no. 10, pp. 2115-2124, 2019.

[18] M. Kanatsu-Shinohara, H. Morimoto, and T. Shinohara, "Fertility of male germline stem cells following spermatogonial transplantation in infertile mouse models," Biology of Reproduction, vol. 94, no. 5, p. 112, 2016. 
[19] H. Zhu, C. Liu, M. Li, J. Sun, W. Song, and J. Hua, “Optimization of the conditions of isolation and culture of dairy goat male germline stem cells (mGSC)," Animal Reproduction Science, vol. 137, no. 1-2, pp. 45-52, 2013.

[20] S. Takashima and T. Shinohara, "Culture and transplantation of spermatogonial stem cells," Stem Cell Research, vol. 29, pp. $46-55,2018$.

[21] H. Zhu, J. Ma, R. du et al., "Characterization of immortalized dairy goat male germline stem cells (mGSCs)," Journal of Cellular Biochemistry, vol. 115, no. 9, pp. 1549-1560, 2014.

[22] H. Kubota, M. R. Avarbock, and R. L. Brinster, "Growth factors essential for self-renewal and expansion of mouse spermatogonial stem cells," Proceedings of the National Academy of Sciences of the United States of America, vol. 101, no. 47, pp. 16489-16494, 2004.

[23] Y. Zhang, S. Wang, X. Wang, S. Liao, Y. Wu, and C. Han, "Endogenously produced FGF2 is essential for the survival and proliferation of cultured mouse spermatogonial stem cells," Cell Research, vol. 22, no. 4, pp. 773-776, 2012.

[24] T. Ogawa, J. M. Aréchaga, M. R. Avarbock, and R. L. Brinster, "Transplantation of testis germinal cells into mouse seminiferous tubules," The International Journal of Developmental Biology, vol. 41, no. 1, pp. 111-122, 1997.

[25] M. Kanatsu-Shinohara, N. Ogonuki, K. Inoue, A. Ogura, S. Toyokuni, and T. Shinohara, "Restoration of fertility in infertile mice by transplantation of cryopreserved male germline stem cells," Human Reproduction, vol. 18, no. 12, pp. 2660-2667, 2003.

[26] S. Goodyear and R. Brinster, "Spermatogonial stem cell transplantation to the testis," Cold Spring Harbor Protocols, vol. 2017, no. 4, 2017.

[27] Z. du, S. Xu, S. Hu et al., "Melatonin attenuates detrimental effects of diabetes on the niche of mouse spermatogonial stem cells by maintaining Leydig cells," Cell Death \& Disease, vol. 9, no. 10 , p. ???, 2018.

[28] Q. Lei, Q. Pan, N. Li et al., "H19 regulates the proliferation of bovine male germline stem cells via IGF-1 signaling pathway," Journal of Cellular Physiology, vol. 234, no. 1, pp. 915-926, 2018.

[29] J. Fang, Y. Wei, X. Teng, S. Zhao, and J. Hua, "Immortalization of canine adipose-derived mesenchymal stem cells and their seminiferous tubule transplantation," Journal of Cellular Biochemistry, vol. 119, no. 4, pp. 3663-3670, 2018.

[30] M. Li, C. Liu, H. Zhu et al., "Expression pattern of boule in dairy goat testis and its function in promoting the meiosis in male germline stem cells (mGSCs)," Journal of Cellular Biochemistry, vol. 114, no. 2, pp. 294-302, 2013.

[31] S. M. White, M. L. Avantaggiati, I. Nemazanyy et al., "YAP/TAZ inhibition induces metabolic and signaling rewiring resulting in targetable vulnerabilities in _NF2-_ deficient tumor cells," Developmental Cell, vol. 49, no. 3, pp. 425443.e9, 2019, e9.

[32] L. Li, C. Ge, D. Wang, L. Yu, J. Zhao, and H. Ma, "Dehydroepiandrosterone reduces accumulation of lipid droplets in primary chicken hepatocytes by biotransformation mediated _via_the cAMP/PKA-ERK1/2 signaling pathway," Biochimica et Biophysica Acta - Molecular and Cell Biology of Lipids, vol. 1863, no. 6, pp. 625-638, 2018.

[33] Y. Qin, L. Liu, Y. N. He et al., “Testicular busulfan injection in mice to prepare recipients for spermatogonial stem cell trans- plantation is safe and non-toxic," PLoS One, vol. 11, no. 2, article e0148388, 2016.

[34] G. Borck, B. S. Shin, B. Stiller et al., "eIF2 $\gamma$ mutation that disrupts eIF2 complex integrity links intellectual disability to impaired translation initiation," Molecular Cell, vol. 48, no. 4, pp. 641-646, 2012.

[35] C. Murata, Y. Kuroki, I. Imoto, and A. Kuroiwa, "Ancestral Ylinked genes were maintained by translocation to the $\mathrm{X}$ and $\mathrm{Y}$ chromosomes fused to an autosomal pair in the Okinawa spiny rat Tokudaia muenninki," Chromosome Research, vol. 24, no. 3, pp. 407-419, 2016.

[36] Z. Niu, L. Zheng, S. Wu et al., "Ras/ERK1/2 pathway regulates the self-renewal of dairy goat spermatogonia stem cells," Reproduction, vol. 149, no. 5, pp. 445-452, 2015.

[37] K. Hasegawa, S. H. Namekawa, and Y. Saga, "MEK/ERK signaling directly and indirectly contributes to the cyclical selfrenewal of spermatogonial stem cells," Stem Cells, vol. 31, no. 11, pp. 2517-2527, 2013.

[38] S. Moortgat, J. Désir, V. Benoit et al., "Two novel EIF2S3 mutations associated with syndromic intellectual disability with severe microcephaly, growth retardation, and epilepsy," American Journal of Medical Genetics. Part A, vol. 170, no. 11, pp. 2927-2933, 2016.

[39] M. Skopkova, F. Hennig, B. S. Shin et al., "EIF2S3 mutations associated with severe X-linked intellectual disability syndrome MEHMO," Human Mutation, vol. 38, no. 4, pp. 409$425,2017$.

[40] S. K. Young-Baird, B. S. Shin, and T. E. Dever, "MEHMO syndrome mutation EIF2S3-I259M impairs initiator MettRNAiMet binding to eukaryotic translation initiation factor eIF2," Nucleic Acids Research, vol. 47, no. 2, pp. 855-867, 2019.

[41] E. A. Ortega, Q. Salvador, M. Fernandez, and M. A. Ward, "Alterations of sex determination pathways in the genital ridges of males with limited Y chromosome genes $\dagger$," Biology of Reproduction, vol. 100, no. 3, pp. 810-823, 2019. 